\title{
Fast Tensor Product Solvers for Optimization Problems with Fractional Differential Equations as Constraints
}

\author{
Sergey Dolgova, John W. Pearson ${ }^{\mathrm{b}}$, Dmitry V. Savostyanov ${ }^{\mathrm{c}}$, Martin Stolla \\ ${ }^{a}$ Numerical Linear Algebra for Dynamical Systems, Max Planck Institute for Dynamics of Complex \\ Technical Systems, Sandtorstr. 1, 39106 Magdeburg, Germany \\ ${ }^{b}$ School of Mathematics, Statistics and Actuarial Science, University of Kent, Cornwallis Building (East), \\ Canterbury, Kent, CT2 7NZ, United Kingdom \\ ${ }^{c}$ School of Computing, Engineering and Mathematics, University of Brighton, Moulsecoomb, Brighton, BN2 \\ 4GJ, United Kingdom
}

\begin{abstract}
Fractional differential equations have recently received much attention within computational mathematics and applied science, and their numerical treatment is an important research area as such equations pose substantial challenges to existing algorithms. An optimization problem with constraints given by fractional differential equations is considered, which in its discretized form leads to a high-dimensional tensor equation. To reduce the computation time and storage, the solution is sought in the tensor-train format. We compare three types of solution strategies that employ sophisticated iterative techniques using either preconditioned Krylov solvers or tailored alternating schemes. The competitiveness of these approaches is presented using several examples with constant and variable coefficients.
\end{abstract}

Keywords: Fractional calculus, Iterative solvers, Sylvester equations, Preconditioning, Low-rank methods, Tensor equations, Schur complement

\section{Introduction}

While the study of derivatives of arbitrary order is a long-standing subject area [30], its use in science and engineering has soared over recent years. Fractional calculus is often used due to the inadequateness of traditional schemes to describe certain phenomena, such as anomalous diffusion, anaelasticity [16] and viscoelasticity [44, 88]. The applications include electrical circuits [39, 73], electro-analytical chemistry [84], biomechanics [27], and image processing [91].

Over the last decade many researchers have worked on efficient numerical schemes for the discretization and solution of fractional differential equations (FDEs). Historically, the finite difference-based discretization techniques are arguably the most popular [50, 51, 71, 72, 73]. Adomian decomposition should be mentioned as a popular semi-analytical approach [1], although its use is limited. Recently, (discontinuous) finite element schemes for FDEs have also received considerable attention [17, 58, 90]. Since the fractional differential operators

Email addresses: dolgov@mpi-magdeburg.mpg.de (Sergey Dolgov), J.W.Pearson@kent.ac.uk (John

W. Pearson), d.savostyanov@brighton.ac.uk (Dmitry V. Savostyanov), stollm@mpi-magdeburg.mpg.de (Martin Stoll)

${ }^{1}$ Tel.: +493916110 450; fax: +493916110453 
are, in fact, integral operators, the matrix of the corresponding linear system is usually dense, and the numerical complexity and storage grow rapidly with the grid size, especially for the problems posed in higher dimensions (e.g. three spatial plus a temporal dimension). To perform computations faster, we compress the solution in the low-rank format. This approach has already been applied to fractional calculus in [13, 75].

Most commonly, the fractional calculus literature focuses on the solution of the equation itself, the so-called 'direct problem'. In this paper we consider the 'inverse problem', namely the computation of the forcing term (right-hand side of the FDE), that is best suited to describing a desired property or measured data. For this we study an optimization problem with constraints given by FDEs. In the context of partial differential equations (PDEs), problems of this type are often referred to as PDE-constrained optimization problems and have been studied extensively over the last decades (see [37, 85] for introductions to the field). Optimal control problems for FDEs have previously been studied in literature such as [2, 3, 54, 55, 65, 74]. However, they were mostly considering one-dimensional spatial domains, since the direct treatment of higher dimensions was too expensive. To overcome computational challenges, in this paper we rely on the recent advances in the development of numerical algorithms and solvers, particularly on data-sparse low-rank formats.

The goal of our paper is to present efficient numerical methods that allow the fast and accurate solution of the large optimization problem at hand. The paper is organized as follows. In Section 2.1 we recall some of the most important definitions needed for fractional derivatives. This is followed by Section 2.2 where we introduce the basic optimization problem subject to fractional differential equations posed in an increasing number of dimensions, along with the discretization of both the objective function and the differential equation. Section

3 presents three strategies that are well-suited to solving the discretized problem. This is followed by a discussion of numerical algorithms in Section 4 where we introduce the tensor-train format and several iterative solvers, either of Krylov subspace type or using an alternating framework. The effectiveness of our approach is shown in Section 5 where we compare our solvers using several numerical experiments. We also present results for the more challenging variable coefficient case and observe satisfying results.

Another approach that has recently been studied by Burrage et al. is to consider a matrix function approach to solve the discretized system (see [15] for details). Our work here is motivated by some recent results in [72] where the discretization via finite differences is considered in a purely algebraic framework.

\section{Fractional calculus and Grünwald formulae}

In this section we briefly recall the concept of fractional derivatives, and use this to state the matrix systems that result from discretizing the problems we consider using a finite difference method. The literature on fractional derivatives is vast and we refer to [18, 30, 34, 53, 69, 70] for general introductions to this topic.

\subsection{The fractional derivative}

In fractional calculus there are several definitions of fractional derivatives. The Caputo and the Riemann-Liouville fractional derivatives [69] are among the most commonly used in applications and we use this section to briefly recall their definitions. 
For a function $f(t)$ defined on an interval $[a, b]$, the Caputo derivative of real order $\alpha$ with $n-1<\alpha<n, n \in \mathbb{N}$, is defined as the following integral

$$
{ }_{a}^{\mathrm{C}} D_{t}^{\alpha} f(t)=\frac{1}{\Gamma(n-\alpha)} \int_{a}^{t} \frac{d^{n} f(s)}{d s^{n}} \frac{d s}{(t-s)^{\alpha-n+1}},
$$

assuming that it is convergent (see [19, 30, 50, 69, 78] for more details). Based on the discussion in [72, the Caputo derivative is frequently used for the derivative with respect to time. The left-sided Riemann-Liouville derivative of real order $\alpha$ with $n-1<\alpha<n$, is defined by

$$
{ }_{a}^{\mathrm{RL}} D_{t}^{\alpha} f(t)=\frac{1}{\Gamma(n-\alpha)} \frac{d^{n}}{d t^{n}} \int_{a}^{t} \frac{f(s) d s}{(t-s)^{\alpha-n+1}},
$$

for $a<t<b$. The right-sided Riemann-Liouville fractional derivative is given by

$$
{ }_{t}^{\mathrm{RL}} D_{b}^{\alpha} f(t)=\frac{(-1)^{n}}{\Gamma(n-\alpha)} \frac{d^{n}}{d t^{n}} \int_{t}^{b} \frac{f(s) d s}{(s-t)^{\alpha-n+1}},
$$

for $a<t<b$. Finally, the symmetric Riesz derivative of order $\alpha$ is the half-sum of the left and right-side Riemann-Liouville derivative, i.e.,

$$
{ }^{\mathrm{R}} D_{t}^{\alpha} f(t)=\frac{1}{2}\left({ }_{a}^{\mathrm{RL}} D_{t}^{\alpha} f(t)+{ }_{t}^{\mathrm{RL}} D_{b}^{\alpha} f(t)\right) .
$$

In this work we do not advocate a particular method that is most suitable for the description of a natural phenomenon: we simply want to illustrate that the above formulations of FDEs, when coupled with certain types of discretization approaches, lead to similar structures on the discrete level. Our goal is to give guidelines and offer numerical schemes for the efficient and accurate solution of problems of various forms. For a discussion on the smoothness assumptions of the function $f(\cdot)$ we refer to [69], i.e., the Riemann-Liouville formulation requires a weaker differentiability assumption in contrast to the $n$-times differentiability required otherwise (cf. [69, Chapter 2.3]).

\subsection{Model problems}

In this section we introduce some FDE-constrained optimization problems. Consider the classical misfit problem, where we want to minimize the difference between the state $y$ and the desired state (or observation) $\bar{y}$, with an additional regularization representing the cost

of the control $u$. The constraint linking the state to the control is given by the following FDE

$$
\begin{aligned}
\left({ }_{0}^{\mathrm{C}} D_{t}^{\alpha}-{ }^{\mathrm{R}} D_{x}^{\beta}\right) y(x, t)+u(x, t) & =f(x, t), \\
\left({ }_{0}^{\mathrm{C}} D_{t}^{\alpha}-{ }^{\mathrm{R}} D_{x_{1}}^{\beta_{1}}-{ }^{\mathrm{R}} D_{x_{2}}^{\beta_{2}}\right) y\left(x_{1}, x_{2}, t\right)+u\left(x_{1}, x_{2}, t\right) & =f\left(x_{1}, x_{2}, t\right), \\
\left({ }_{0}^{\mathrm{C}} D_{t}^{\alpha}-{ }^{\mathrm{R}} D_{x_{1}}^{\beta_{1}}-{ }^{\mathrm{R}} D_{x_{2}}^{\beta_{2}}-{ }^{\mathrm{R}} D_{x_{3}}^{\beta_{3}}\right) y\left(x_{1}, x_{2}, x_{3}, t\right)+u\left(x_{1}, x_{2}, x_{3}, t\right) & =f\left(x_{1}, x_{2}, x_{3}, t\right)
\end{aligned}
$$

in one, two, and three dimensions, respectively. We consider the FDEs in space-time cylinder domains $Q:=\Omega \times[0, T]$, with $\Omega \subseteq \mathbb{R}^{\bar{d}}, \bar{d} \in\{1,2,3\}$. We also denote $\operatorname{supp}(\bar{y})=Q_{\bar{y}}$ and $\operatorname{supp}(u)=Q_{u}$ and assume that both the observation and the control are supported over the cylinders $Q_{\bar{y}}=\Omega_{\bar{y}} \times[0, T]$ and $Q_{u}=\Omega_{u} \times[0, T]$. Our cost function is, therefore

$$
J(y, u):=\frac{1}{2} \iint_{Q_{\bar{y}}}(y-\bar{y})^{2} d x d t+\frac{\gamma}{2} \iint_{Q_{u}} u^{2} d x d t
$$


where $\gamma$ is a regularization parameter indicating at what ratio one prioritizes minimizing $u$, and obtaining $y$ that is close in some sense to the desired state $\bar{y}$.

We will follow the discretize-then-optimize approach for PDE-constrained optimization problems [11, 35] (details on the numerical treatment of FDEs can be found in [50, 51, 69, [71, 72]). We consider $\Omega=[0,1]^{\bar{d}}$ and assume that $\Omega_{u}$ and $\Omega_{\bar{y}}$ are also cubes.

Each function is discretized by collocation on a uniform tensor product grid in space and time

$$
\begin{gathered}
x_{k}\left(i_{k}\right)=i_{k} h_{k}, \quad i_{k}=1, \ldots, n_{k}, \quad h_{k}=\frac{1}{n_{k}+1}, \quad k=1,2,3, \\
t(m)=m \tau, \quad m=1, \ldots, n_{t}, \quad \tau=\frac{T}{n_{t}} .
\end{gathered}
$$

After discretization, vectors containing values of functions $y, \bar{y}, u, f$ on the grid are denoted also as $y, \bar{y}, u, f$, respectively. We assume zero boundary and initial conditions, $y\left(x_{1}, \ldots, x_{\bar{d}}, t\right)=0$ if $x_{k} \leq 0, x_{k} \geq 1$ or $t \leq 0$. Note that it is possible to include inhomogeneous boundary and initial conditions [49, 69]. This typically does not affect the matrix structure and so the methods presented here also apply for this setup. If the grid sizes are the same in all spatial directions, we denote $h=h_{1}=h_{2}=h_{3}$.

Note that our formulations here are tailored to domains that allow for tensorized grids, as we aim to separate the variables with respect to all spatial and temporal dimensions. While this does exclude certain applications, many interesting and relevant models can still be considered such as fractional phase-field models [12, 14, 15] or the fractional FokkerPlanck equation [48. They also appear in many applications such as the anomalous diffusion [52], pattern formation using fractional derivatives [28, and also the simulation of fractional phase-field equations such as the Allen-Cahn equation [15]. Additionally, the low-rank approximation presented in this paper also applies in the case where the spatial domain is more complicated. In this case one can often still separate spatial and temporal dimensions, or other parameter dimensions, which are often found for problems with uncertainties and/or model parameters.

\subsection{Grünwald-Letnikov formula}

A classical method for the discretization of FDEs is based on the formula of Grünwald and Letnikov here illustrated for the one-dimensional case. In particular, for the spatial derivative we use its shifted version

$$
{ }_{0}^{\mathrm{RL}} D_{x}^{\beta} y(x) \approx \frac{1}{h^{\beta}} \sum_{i=0}^{n} g_{\beta, i} y(x-(i-1) h)
$$

which is proven to be of the first order [49, 50, 51], provided that $y \in W_{1}^{1+\beta}(\mathbb{R})$. The coefficients $g_{\beta, i}$ are defined by

$$
g_{\beta, i}=\frac{\Gamma(i-\beta)}{\Gamma(-\beta) \Gamma(i+1)}=(-1)^{i}\left(\begin{array}{c}
\beta \\
i
\end{array}\right),
$$

and can be computed efficiently using the recurrent formula [69]: $g_{\beta, 0}=1, g_{\beta, i}=\left(1-\frac{\beta+1}{i}\right) g_{\beta, i-1}$ for $i=1,2, \ldots, n$. 
Collecting all degrees of freedom into one matrix, we obtain the discretization of the spatial Riemann-Liouville derivative as follows

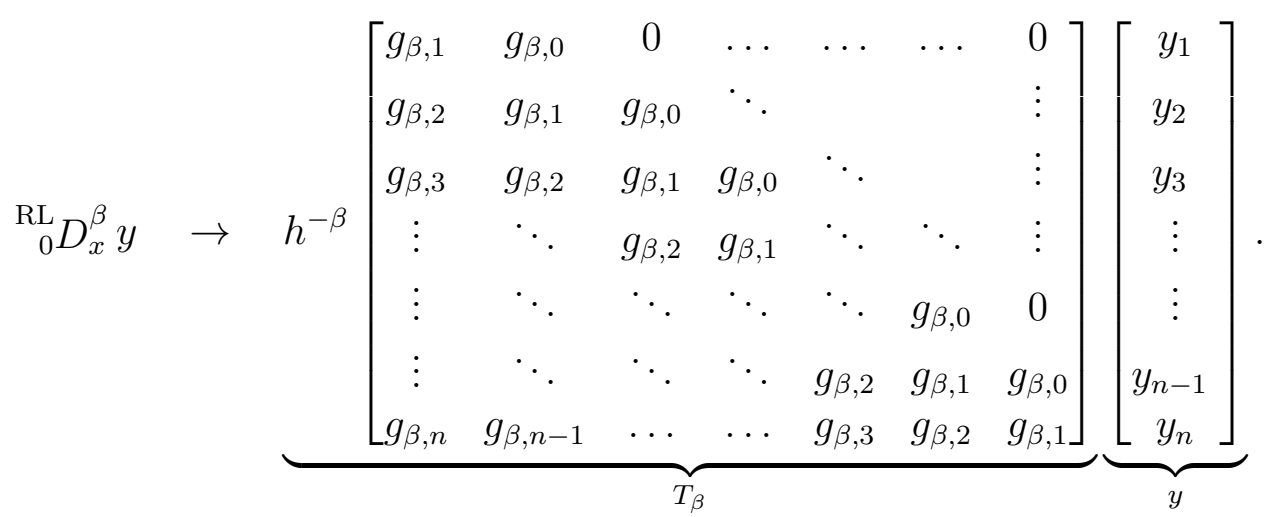

The matrix $T_{\beta}$ is a Toeplitz matrix, which we discuss later in more detail. As in [72] we approximate the spatial derivative of order $1 \leq \beta \leq 2$ using the symmetric Riesz derivative taken as the half sum of the left- and right-sided Riemann-Liouville fractional derivatives. The resulting differentiation matrix is given by

$$
L_{\beta}:=\frac{1}{2}\left(T_{\beta}+T_{\beta}^{\top}\right) .
$$

The discretization in time is done via the non-shifted Grünwald-Letnikov formula [69, 78]

$$
{ }_{0}^{\mathrm{C}} D_{t}^{\alpha} y(t) \approx \frac{1}{\tau^{\alpha}} \sum_{m=0}^{n_{t}-1} g_{\alpha, m} y(t-m \tau),
$$

and the Caputo derivative leads to a Toeplitz matrix $C_{\alpha}$ of the lower triangular form

$$
C_{\alpha}=\tau^{-\alpha}\left[\begin{array}{ccccccc}
g_{\alpha, 0} & 0 & \cdots & \cdots & \ldots & \ldots & 0 \\
g_{\alpha, 1} & g_{\alpha, 0} & \ddots & & & & \vdots \\
g_{\alpha, 2} & g_{\alpha, 1} & g_{\alpha, 0} & \ddots & & & \vdots \\
\vdots & \ddots & g_{\alpha, 1} & g_{\alpha, 0} & \ddots & & \vdots \\
\vdots & \ddots & \ddots & \ddots & \ddots & \ddots & \vdots \\
\vdots & \ddots & \ddots & \ddots & g_{\alpha, 1} & g_{\alpha, 0} & 0 \\
g_{\alpha, n_{t}-1} & g_{\alpha, n_{t}-2} & \cdots & \cdots & g_{\alpha, 2} & g_{\alpha, 1} & g_{\alpha, 0}
\end{array}\right]
$$

\subsection{Problem structure}

Since the spatial grids introduced on $\Omega, \Omega_{\bar{y}}$ and $\Omega_{u}$ have Cartesian product structure, e.g. these domains are cubes in $\mathbb{R}^{\bar{d}}$, the discretized differential operators in $(1 \mathrm{~b})$ and $(1 \mathrm{c})$ can be written using the tensor-product notation,

$$
\begin{aligned}
{ }^{\mathrm{R}} D_{x_{1}}^{\beta_{1}}+{ }^{\mathrm{R}} D_{x_{2}}^{\beta_{2}} & \rightarrow \quad L=L_{\beta_{1}} \otimes I_{n_{2}}+I_{n_{1}} \otimes L_{\beta_{2}}, \\
{ }^{\mathrm{R}} D_{x_{1}}^{\beta_{1}}+{ }^{\mathrm{R}} D_{x_{2}}^{\beta_{2}}+{ }^{\mathrm{R}} D_{x_{3}}^{\beta_{3}} & \rightarrow \quad L=L_{\beta_{1}} \otimes I_{n_{2}} \otimes I_{n_{3}}+I_{n_{1}} \otimes L_{\beta_{2}} \otimes I_{n_{3}}+I_{n_{1}} \otimes I_{n_{2}} \otimes L_{\beta_{3}},
\end{aligned}
$$

for $\bar{d}=2$ and $\bar{d}=3$, respectively. Here and later $I_{n}$ denotes an identity matrix of size $n$. 
Assuming that $L$ denotes the discretization of spatial derivatives (9), we obtain the discretization of the FDEs as follows

$$
A y+M_{3} u=g, \quad A=C_{\alpha} \otimes I_{n}-I_{n_{t}} \otimes L,
$$

where $n$ denotes the total number of space mesh points, $M_{3} u$ is the discretization of the control term, and $g$ represents boundary conditions, initial conditions and additional forcing terms. Note that vectors $y$ and $g$ are of the size $N=n n_{t}$, where $n=n_{1} n_{2}$ for a twodimensional problem and $n=n_{1} n_{2} n_{3}$ for a three-dimensional problem. The vector $u$ is formally of a smaller size, since we only consider its non-zero elements, which are all on $Q_{u} \subseteq Q$. Denoting the numbers of grid points on $\Omega_{u}$ as $n^{\prime}$, and hence the number of grid points in $Q_{u}$ is $N^{\prime}=n^{\prime} n_{t}$, we conclude that $M_{3}$ is the $N \times N^{\prime}$ matrix. In our case of the collocation discretization, $M_{3}$ contains unitary rows at positions, corresponding to the grid points in $Q_{u}$.

Using standard integration rules, we discretize the cost function (2) as follows

$$
J(y, u)=\frac{1}{2}(y-\bar{y})^{\top} M_{1}(y-\bar{y})+\frac{\gamma}{2} u^{\top} M_{2} u,
$$

where $M_{1}$ and $M_{2}$ contain the quadrature weights used to evaluate the norm. We use the trapezoidal rule, coinciding with the rectangular rule due to zero boundary conditions. We make the vector $\bar{y}$ of size $N$. Since the function $\bar{y}\left(x_{1}, x_{2}, x_{3}, t\right)$ is defined only on $\Omega_{\bar{y}}$, we populate $\bar{y}$ by zeros at the remaining points, lying in $\Omega \backslash \Omega_{\bar{y}}$. Let us denote by $\theta_{\bar{y}} \in \mathbb{R}^{N}$ a binary vector, containing 1's at indices belonging to $\Omega_{\bar{y}}$. Then

$$
M_{1}=\tau h_{1} \cdots h_{\bar{d}} \cdot \operatorname{diag}\left(\theta_{\bar{y}}\right), \quad \text { and } \quad M_{2}=\tau h_{1} \cdots h_{\bar{d}} \cdot I_{N^{\prime}} .
$$

A standard Lagrangian approach results in the following discrete functional for which we need to compute stationary points:

$$
\Lambda(y, u, p)=J(y, u)+p^{\top} V^{\top}\left(A y+M_{3} u-g\right),
$$

where $p$ corresponds to the discretized adjoint variable $p$. The matrix $V$ is used to associate $p$ with a grid function (see [1] ), and is written similarly to (12), $V=\tau h_{1} \cdots h_{\bar{d}} \cdot I_{N}$. To obtain the required optimality conditions, we differentiate $\Lambda$ with respect to $y, u$ and $p$. This leads to the following first order, Karush-Kuhn-Tucker (KKT), system:

$$
\underbrace{\left[\begin{array}{ccc}
M_{1} & 0 & A^{\top} V \\
0 & \gamma M_{2} & M_{3}^{\top} V \\
V^{\top} A & V^{\top} M_{3} & 0
\end{array}\right]}_{\mathcal{A}}\left[\begin{array}{l}
y \\
u \\
p
\end{array}\right]=\left[\begin{array}{c}
M_{1} \bar{y} \\
0 \\
V^{\top} g
\end{array}\right]=:\left[\begin{array}{l}
f_{1} \\
f_{2} \\
f_{3}
\end{array}\right],
$$

For simplicity, we merge $V$ into both $A$ and $M_{3}$ and proceed with the following system matrix:

$$
\mathcal{A}=\left[\begin{array}{ccc}
M_{1} & 0 & A^{\top} \\
0 & \gamma M_{2} & M_{3}^{\top} \\
A & M_{3} & 0
\end{array}\right]
$$

The invertibility of the matrix follows from the constraint block $\left[\begin{array}{ll}A & M_{3}\end{array}\right]$ having full-rank, and the fact that the $(1,1)$-block is positive definite on the kernel of the constraints, i.e.,

$$
\left[\begin{array}{ll}
-u^{\top} M_{3}^{\top} A^{-\top} & u^{\top}
\end{array}\right]\left[\begin{array}{cc}
M_{1} & 0 \\
0 & \gamma M_{2}
\end{array}\right]\left[\begin{array}{c}
-A^{-1} M_{3} u \\
u
\end{array}\right]=u^{\top} M_{3}^{\top} A^{-\top} M_{1} A^{-1} M_{3} u+\gamma u^{\top} M_{2} u>0
$$

(see [10]). There are various ways to solve the saddle point system and in the next section we discuss several approaches. 


\section{Solution strategies}

In this section we discuss possible methods to solve the system (14). The classical monolithic approach is not applicable in our case: a direct solver is prohibitively expensive due to the problem size. Therefore, we consider three types of iterative methods: a low-rank MinRES method applied to (14) and alternating iterative methods applied to two variants of Schur complements.

\subsection{Krylov solver and preconditioning}

For linear systems the use of iterative methods of Krylov type [77] is well established. For standard saddle point problems in particular this is a method of choice, and is considered a state of the art when the problem is of very high dimension (see [10, 25]). Such a solver proceeds by building up a Krylov subspace

$$
\mathcal{K}_{l}\left(\mathcal{A}, r_{0}\right)=\operatorname{span}\left\{r_{0}, \mathcal{A} r_{0}, \ldots, \mathcal{A}^{l-1} r_{0}\right\},
$$

in terms of the initial residual vector $r_{0}$. The solution at step $l$ is then computed in some (quasi-)optimal way within the $\operatorname{Krylov}$ subspace $\mathcal{K}_{l}\left(\mathcal{A}, r_{0}\right)$.

As the convergence can sometimes be very slow the problem is modified using a preconditioning matrix $\mathcal{P}$, i.e.,

$$
\mathcal{A} y=f \quad \Leftrightarrow \quad \mathcal{P}^{-1} \mathcal{A} y=\mathcal{P}^{-1} f .
$$

We again refer to [10, 25] for a detailed overview of preconditioners for saddle point problems. In this paper, we follow a result presented in [56], where it was shown that for a saddle point system

$$
\left[\begin{array}{cc}
\Phi & \Psi^{\top} \\
\Psi & 0
\end{array}\right] y=f
$$

a good preconditioner can be chosen in the form

$$
\mathcal{P}=\left[\begin{array}{cc}
\widetilde{\Phi} & 0 \\
0 & \widetilde{S}
\end{array}\right] \quad \text { or } \quad \mathcal{P}=\left[\begin{array}{cc}
\widetilde{\Phi} & 0 \\
\Psi & -\widetilde{S}
\end{array}\right]
$$

where $\widetilde{\Phi} \approx \Phi$ and $\widetilde{S} \approx S:=\Psi \Phi^{-1} \Psi^{\top}$, the (negative) Schur complement. For our problem, we commence with the approximation of the following $(1,1)$-block:

$$
\Phi=\left[\begin{array}{cc}
M_{1} & 0 \\
0 & \gamma M_{2}
\end{array}\right] .
$$

In the case of the full observation and control, $\Omega_{\bar{y}}=\Omega_{u}=\Omega$, all $M_{i}, i=1,2,3$, are simply scaled identity matrices. They can be easily inverted, so $\Phi$ can be "approximated" exactly. For the Schur complement $S=\Psi \Phi^{-1} \Psi^{\top}$ with the constraint matrix $\Psi=\left[\begin{array}{ll}A & M_{3}\end{array}\right]$ it is typically much harder to find a robust approximation. For this we study the structure of the Schur complement further:

$$
S=A M_{1}^{-1} A^{\top}+\frac{1}{\gamma} M_{3} M_{2}^{-1} M_{3}^{\top} .
$$

The authors have recently obtained robust Schur complement approximations using a matching approach [66, 67, 83] that in our case leads to

$$
\widetilde{S}=\widetilde{A} M_{1}^{-1} \widetilde{A}^{\top}, \quad \widetilde{A}=A+\frac{1}{\sqrt{\gamma}} I_{n_{t}} \otimes I_{n} .
$$


Before discussing the efficient inversion of the matrix $\widetilde{A}$, we briefly analyze the effectiveness of our Schur complement approximation. It is clear that the effectiveness of our solver for the matrix system depends to a large extent on how well the exact Schur complement as given in (15) is represented by our approximation (16). Following [68], we measure this by examining the Rayleigh quotient

$$
R:=\frac{v^{\top} S v}{v^{\top} \widetilde{S} v}, \quad v \in \mathbb{R}^{N}, \quad v \neq 0 .
$$

Theorem 1. Consider the FDE-constrained control problem (1)-(2) with Grünwald-Letnikov discretizations (6), (8) on the same cubic domain $\Omega=\Omega_{\bar{y}}=\Omega_{u}$ for the observation, control and system state, with the simplifying assumption that $M_{1}=M_{2}=M_{3}$ are scaled mass matrices. Let $0<\alpha \leq 1$ and $1 \leq \beta_{i} \leq 2, i=1,2,3$. Then it holds $\frac{1}{2} \leq R<1$.

Proof. Following [66, we write

$$
R=\frac{a_{1}^{\top} a_{1}+a_{2}^{\top} a_{2}}{\left(a_{1}+a_{2}\right)^{\top}\left(a_{1}+a_{2}\right)},
$$

where $a_{1}=\frac{1}{\sqrt{\tau h^{\bar{d}}}}\left(C_{\alpha}^{\top} \otimes I_{n}-I_{n_{t}} \otimes L^{\top}\right) v, a_{2}=\sqrt{\frac{\tau h^{\bar{d}}}{\gamma}} v$, using the form of $M_{1}, M_{2}, M_{3}$. We first note that $a_{2}^{\top} a_{2}=\frac{\tau h^{\bar{d}}}{\gamma} v^{\top} v>0$. This means that we may write

$$
\frac{1}{2}\left(a_{1}-a_{2}\right)^{\top}\left(a_{1}-a_{2}\right) \geq 0 \quad \Leftrightarrow \quad a_{1}^{\top} a_{1}+a_{2}^{\top} a_{2} \geq \frac{1}{2}\left(a_{1}+a_{2}\right)^{\top}\left(a_{1}+a_{2}\right) \quad \Leftrightarrow \quad R \geq \frac{1}{2} .
$$

To prove the upper bound for $R$, we now consider the quantity

$$
a_{1}^{\top} a_{2}+a_{2}^{\top} a_{1}=\frac{1}{\sqrt{\gamma}} v^{\top}\left(\left(C_{\alpha}+C_{\alpha}\right)^{\top} \otimes I_{n}-I_{n_{t}} \otimes\left(L+L^{\top}\right)\right) v .
$$

It has been shown in [13, Lemma 1] that the matrix $I_{n_{t}} \otimes L$ is negative semi-definite for $1 \leq \beta_{i} \leq 2$ - we can therefore write that $v^{\top}\left(I_{n_{t}} \otimes\left(L+L^{\top}\right)\right) v=2 v^{\top}\left(I_{n_{t}} \otimes L\right) v \leq 0$, using the symmetry of $L$.

The matrix $C_{\alpha}+C_{\alpha}^{\top}$ is symmetric positive definite for $\alpha<1$, since $C_{\alpha}$ is diagonally dominant. It is clear that the coefficient $g_{\alpha, k}$ is positive for $k=0$ and negative for all $k=1,2, \ldots$, if $\alpha<1$, so we need to show that $\sum_{k=0}^{n_{t}} g_{\alpha, k}>0$. This follows from the binomial expansion $(1+z)^{\alpha}=\sum_{k=0}^{\infty}\left(\begin{array}{l}\alpha \\ k\end{array}\right) z^{k}$, evaluated at $z=-1$. In our case, Re $\alpha>0$ and $|z|=1$, and this series converges absolutely [33, page 397]. Therefore, the following inequality holds, $\sum_{k=0}^{n_{t}} g_{\alpha, k}>\sum_{k=0}^{\infty} g_{\alpha, k}=\sum_{k=0}^{\infty}\left(\begin{array}{l}\alpha \\ k\end{array}\right)(-1)^{k}=0$. Hence, we have that $v^{\top}\left(\left(C_{\alpha}+C_{\alpha}^{\top}\right) \otimes I_{n}\right) v>$ 0 , using the standard property of Kronecker product that the eigenvalues of $K_{1} \otimes K_{2}$ are equal to those of $K_{1}$ multiplied by those of $K_{2}$ [47, Chapter 13].

Combining these findings gives us that $a_{1}^{\top} a_{2}+a_{2}^{\top} a_{1}>0$. We therefore have that

$$
R=\frac{a_{1}^{\top} a_{1}+a_{2}^{\top} a_{2}}{a_{1}^{\top} a_{1}+a_{2}^{\top} a_{2}+a_{1}^{\top} a_{2}+a_{2}^{\top} a_{1}}<\frac{a_{1}^{\top} a_{1}+a_{2}^{\top} a_{2}}{a_{1}^{\top} a_{1}+a_{2}^{\top} a_{2}}=1 .
$$

The general case of incomplete observation or control is analytically much more challenging, but there are heuristic techniques [66, 67] for augmenting the matrices $M_{1}$ and $M_{3}$ which define fairly good (from computational experience) approximations $\widetilde{\Phi}$ and $\widetilde{S}$. 


\subsection{First Schur Complement}

This approach requires the invertibility of the $(1,1)$-block $\Phi$ and is mainly concerned with inverting the (negative) Schur complement matrix

$$
S=A M_{1}^{-1} A^{\top}+M_{3}\left(\gamma M_{2}\right)^{-1} M_{3}^{\top} .
$$

It proceeds by solving the following three systems,

$$
\begin{aligned}
-S p & =f_{3}-A M_{1}^{-1} f_{1}-M_{3}\left(\gamma M_{2}\right)^{-1} f_{2}, \\
\left(\gamma M_{2}\right) u & =f_{2}-M_{3}^{\top} p, \\
M_{1} y & =f_{1}-A^{\top} p .
\end{aligned}
$$

The disadvantage of this approach is that it requires the invertibility of both observation and control matrices for the existence of $S$, which may be restrictive. However, when the matrices $M_{1}$ and $M_{2}$ are invertible, their inversion is usually very cheap, e.g. $M_{1}$ and $M_{2}$ are scaled identities. As we observe through numerical experiments, this scheme is superior when both observation and control are defined on the whole domain.

\subsection{Second Schur Complement}

Now we consider the case when control and observation domains have different sizes, so that $M_{3}$ is a rectangular matrix. In contrast to $M_{1}$, the matrix $M_{2}$ can be assumed to be non-singular, since it corresponds to the regularization term. Therefore, the following decomposition can be verified straightforwardly:

$$
\left[\begin{array}{ccc}
M_{1} & 0 & A^{\top} \\
0 & \gamma M_{2} & M_{3}^{\top} \\
A & M_{3} & 0
\end{array}\right]=\left[\begin{array}{ccc}
I & -M_{1} A^{-1} M_{3} & M_{1} \\
0 & \gamma M_{2} & 0 \\
0 & 0 & A
\end{array}\right]\left[\begin{array}{ccc}
0 & 0 & M_{1} A^{-1} M_{3}\left(\gamma M_{2}\right)^{-1} M_{3}^{\top}+A^{\top} \\
0 & I & \left(\gamma M_{2}\right)^{-1} M_{3}^{\top} \\
I & A^{-1} M_{3} & 0
\end{array}\right] .
$$

The matrix $A$ specifies the underlying FDE model, and is also invertible. Using this factorization the solution of (14) can be computed as follows. First, define

$$
\left[\begin{array}{l}
\tilde{f}_{1} \\
\tilde{f}_{2} \\
\tilde{f}_{3}
\end{array}\right]:=\left[\begin{array}{ccc}
I & -M_{1} A^{-1} M_{3} & M_{1} \\
0 & \gamma M_{2} & 0 \\
0 & 0 & A
\end{array}\right]^{-1}\left[\begin{array}{l}
f_{1} \\
f_{2} \\
f_{3}
\end{array}\right]
$$

We compute these intermediate vectors by solving $A \widetilde{f}_{3}=f_{3},\left(\gamma M_{2}\right) \widetilde{f}_{2}=f_{2}, A g_{2}=M_{3} \widetilde{f}_{2}$ and computing $\widetilde{f}_{1}=f_{1}+M_{1} g_{2}-M_{1} \widetilde{f}_{3}$. Note that if $f_{2}=0$ as in (14), it is trivially observed that $g_{2}=0$. Now we eliminate the second factor in $(19)$ by solving the following systems:

$$
\begin{aligned}
\left(M_{1} A^{-1} M_{3}\left(\gamma M_{2}\right)^{-1} M_{3}^{\top}+A^{\top}\right) p & =\widetilde{f}_{1}, \\
\left(\gamma M_{2}\right) u & =f_{2}-M_{3}^{\top} p, \\
A y & =f_{3}-M_{3} u .
\end{aligned}
$$

Both $M_{2}$ and $A$ have Kronecker-product structures by assumption, hence the action of their inverses by vectors can be computed efficiently using tensor product algorithms. 
The computation of $p$ is more complicated: we have to assemble the Schur complement first, which itself requires matrix inversion. We cast this problem as the solution of a larger linear system: we assemble $\widehat{A}=A \otimes I_{N}$ and solve

$$
\widehat{A} q=\operatorname{vec}\left(M_{3}\left(\gamma M_{2}\right)^{-1} M_{3}^{\top}\right), \quad q=\operatorname{vec}(Q)
$$

where $\operatorname{vec}(\cdot)$ stretches matrix entries to a vector. Now the matrix in 20a becomes $M_{1} Q+A^{\top}$.

In tensor product form, the storage complexity of $\widehat{A}$ is not much higher than that of $A$. A certain difficulty arises from the storage of $Q$ : while $A$ has a convenient tensor product structure, its inversion may consume a considerable amount of memory even in a compressed form. This occurs, for example, if the control is given on the entire domain, and the righthand side in (21) is an identity matrix. Fortunately, in practically interesting cases of a small control domain, the column size of $M_{3}$ is also small. The right-hand side in (21) is a low-rank matrix and yields smaller storage requirements for $Q$ to achieve the same accuracy.

\section{Numerical algorithms}

We will now discuss solvers for the matrix systems $(14)-(21)$. While for the one-dimensional FDE the problem has similarities to saddle point problems involving matrix equations of Sylvester type [81, the higher-dimensional setup requires the use of more specialized tensor solvers. In particular we discuss the tensor-train format introduced in [61].

\subsection{The tensor-train decomposition}

In a one-dimensional problem, where we separate the spatial and time variable, a suitable approach for the FDE problem is a matrix based low-rank decomposition of Krylov vectors. While this is an important case that we discuss in the next section in some more detail, in higher dimensions even low-rank factors become infeasible. Further data compression can be achieved with more advanced high-dimensional tensor product decompositions. In this paper we use the simple, but robust, Tensor Train (TT) format and its extension, the Quantized Tensor Train (QTT) decomposition.

The TT format is derived by applying the low-rank approximation recurrently [61]. Given a $d$-index array $\mathbf{y}=\left[\mathbf{y}\left(i_{1}, \ldots, i_{d}\right)\right]$, with indices varying in ranges $i_{k}=1, \ldots, n_{k}, k=1, \ldots, d$, we may reshape a tensor $\mathbf{y}$ into a matrix $Y_{1} \in \mathbb{R}^{n_{1} \times\left(n_{2} \cdots n_{d}\right)}$ by the grouping of indices. To do this we introduce the notation

$$
\overline{i_{2} \ldots i_{d}}=i_{2}+\left(i_{3}-1\right) n_{2}+\cdots+\left(i_{d}-1\right) n_{2} n_{3} \cdots n_{d-1},
$$

and define $Y_{1}\left(i_{1}, \overline{i_{2} \ldots i_{d}}\right)=\mathbf{y}\left(i_{1}, \ldots, i_{d}\right)$ for all admissible index values. Since $Y_{1}$ is a matrix, we may apply the low-rank singular value decomposition (SVD):

$$
Y_{1} \approx U_{1} \Sigma_{1} V_{1}^{\top}, \quad \text { where } \quad U_{1} \in \mathbb{R}^{n_{1} \times r_{1}}, \quad V_{1} \in \mathbb{R}^{n_{2} \cdots n_{d} \times r_{1}} .
$$

The first factor $U_{1}$ is of moderate dimension, and can be stored as $\mathbf{y}_{\alpha_{1}}^{(1)}\left(i_{1}\right)=U_{1}\left(i_{1}, \alpha_{1}\right)$, where $\alpha_{1}=1, \ldots, r_{1}$. The remaining matrix $\Sigma_{1} V_{1}^{\top}$ depends on indices $\alpha_{1}$ and $\overline{i_{2} \ldots i_{d}}$. Now we regroup these indices as follows:

$$
Y_{2}\left(\overline{\alpha_{1} i_{2}}, \overline{i_{3} \ldots i_{d}}\right)=\Sigma_{1}\left(\alpha_{1}, \alpha_{1}\right) V_{1}^{\top}\left(\alpha_{1}, \overline{i_{2} \ldots i_{d}}\right)
$$


and compute the next SVD,

$$
Y_{2} \approx U_{2} \Sigma_{2} V_{2}^{\top}, \quad \text { where } \quad U_{2} \in \mathbb{R}^{r_{1} n_{2} \times r_{2}}, \quad V_{2} \in \mathbb{R}^{n_{3} \cdots n_{d} \times r_{2}}
$$

Again, $U_{2}$ can be reshaped to a moderately-sized 3-dimensional tensor $\mathbf{y}_{\alpha_{1}, \alpha_{2}}^{(2)}\left(i_{2}\right)=U_{2}\left(\overline{\alpha_{1} i_{2}}, \alpha_{2}\right)$, and the decomposition continued for $\Sigma_{2} V_{2}^{\top}$. Finally, we arrive at the TT format:

$$
\mathbf{y}\left(i_{1}, \ldots, i_{d}\right) \approx \sum_{\alpha_{1}, \ldots, \alpha_{d-1}=1}^{r_{1}, \ldots, r_{d-1}} \mathbf{y}_{\alpha_{1}}^{(1)}\left(i_{1}\right) \mathbf{y}_{\alpha_{1}, \alpha_{2}}^{(2)}\left(i_{2}\right) \cdots \mathbf{y}_{\alpha_{d-2}, \alpha_{d-1}}^{(d-1)}\left(i_{d-1}\right) \mathbf{y}_{\alpha_{d-1}}^{(d)}\left(i_{d}\right)
$$

where $r_{k}$ are called TT ranks. The storage size in the TT format (24) is $\mathcal{O}\left(d n r^{2}\right)$, where $r \gtrsim r_{k}$ and $n \gtrsim n_{k}$.

A similar construction is introduced for discretized operators in high dimensions. Given a matrix $A=\left[A\left(\overline{i_{1} \ldots i_{d}}, \overline{j_{1} \ldots j_{d}}\right)\right] \in \mathbb{R}^{\left(n_{1} \cdots n_{d}\right) \times\left(n_{1} \cdots n_{d}\right)}$, we decompose it as

$$
A\left(\overline{i_{1} \ldots i_{d}}, \overline{j_{1} \ldots j_{d}}\right)=\sum_{\beta_{1}, \ldots, \beta_{d-1}=1}^{R_{1}, \ldots, R_{d-1}} \mathbf{A}_{\beta_{1}}^{(1)}\left(i_{1}, j_{1}\right) \mathbf{A}_{\beta_{1}, \beta_{2}}^{(2)}\left(i_{2}, j_{2}\right) \cdots \mathbf{A}_{\beta_{d-1}}^{(d)}\left(i_{d}, j_{d}\right) \text {, }
$$

which is consistent with the Kronecker product $A=A^{(1)} \otimes A^{(2)}$ in the case $d=2$ and $R_{1}=1$, and allows a natural multiplication with (24), returning the result in the same form.

In the course of computing the matrix-by-vector product $f=A y$, the TT ranks of $A$ and $y$ are multiplied, i.e. $r_{k}(f)=R_{k}(A) r_{k}(y), k=1, \ldots, d-1$. In many cases they are unnecessarily large for achieving the required accuracy. However, as soon as $f$ is already written in the format, its SVD re-compression steps, e.g. (23), can be implemented without high-dimensional tensors appearing. Only QR and SVD decompositions of $n r \times r$ matrices are involved [61]. The total complexity of this procedure is $\mathcal{O}\left(d n r^{3}\right)$, which opens up the possibility of using any standard iterative method, such as MINRES, as soon as $r$ remains moderate during the course of the iterations.

The multi-index concept (22) allows us to compress even "one-dimensional" matrices and vectors, which lack a method for separating variables at first glance. Let us consider $y=[y(i)] \in \mathbb{R}^{n}$, with $n=2^{l}$. Then we may write $i$ in the binary coding,

$$
i=\overline{i_{1} \ldots i_{l}}=i_{1}+2\left(i_{2}-1\right)+\cdots+2^{l-1}\left(i_{l}-1\right), \quad i_{s} \in\{1,2\}, \quad s=1, \ldots, l .
$$

As a result, a vector $y$ is reshaped to an $l$-dimensional tensor $\mathbf{y}$, i.e. $\mathbf{y}\left(i_{1}, \ldots, i_{l}\right)=y(i)$, and the TT approximation can be applied to $\mathbf{y}$. The resulting TT format was called the Quantized TT (QTT) [42, 59, 60, 86]. If the TT ranks of $\mathbf{y}$ are moderate, the total storage reduces to a logarithmic amount $\mathcal{O}\left(l r^{2}\right)=\mathcal{O}(\log n)$.

For many elementary functions and operators, their TT/QTT formats can be written analytically, for example, the discretized Laplace operator [41], the sine, exponential and polynomial functions, sampled on uniform grids in one [42, 62] and many dimensions [20, 43].

The discretisation of fractional order derivatives on a uniform grid is given as a convolution with a vector, which admits a low-rank QTT representation [75]. The corresponding Toeplitz matrix will also have a low QTT rank as shown in [40]. Therefore, the tensor product format can be applied to solve the problem (14).

\subsection{Tensor product Krylov methods}

For expository purposes we start the discussion by considering the case with one spatial and one temporal dimension, which leads to a matrix valued problem. Following this we discuss the more general tensor-valued equation and the corresponding solvers. 
Matrix case

It was recently noted [83] that the vectors $y, u, p \in \mathbb{R}^{n n_{t}}$ for space-time saddle point problems can be written as

$$
\begin{aligned}
& y=\operatorname{vec}(Y)=\operatorname{vec}\left(\left[y_{1}, \ldots, y_{n_{t}}\right]\right), \\
& u=\operatorname{vec}(U)=\operatorname{vec}\left(\left[u_{1}, \ldots, u_{n_{t}}\right]\right), \\
& p=\operatorname{vec}(P)=\operatorname{vec}\left(\left[p_{1}, \ldots, p_{n_{t}}\right]\right) .
\end{aligned}
$$

Note that we can now perform any iterative scheme in matrix form for the unknowns rather than in vector form. This can best be seen by exploiting the identity

$$
\left(-C_{\alpha} \otimes I_{n}+I_{n_{t}} \otimes L\right) \operatorname{vec}(Y)=\operatorname{vec}\left(-I_{n} Y C_{\alpha}^{\top}+L Y I_{n_{t}}^{\top}\right)
$$

and then neglecting the vec operator on the right-hand-side. Additionally one can now use a low-rank decomposition, for instance via the singular value decomposition (SVD), such that $Y=Y_{1} Y_{2}^{\top}, U=U_{1} U_{2}^{\top}$, and $P=P_{1} P_{2}^{\top}$ are the solutions obtained using a low-rank Krylov method. The main ingredients of such a scheme are that the right-hand side and the initial guess are decomposed into low-rank form, which is then maintained throughout the iteration. In more detail, assume that the initial residual is given by

$$
\begin{array}{llll}
R_{Y}=W_{Y} V_{Y}^{\top} & \text { with } & W_{Y} \in \mathbb{R}^{n \times r_{1}}, V_{Y} \in \mathbb{R}^{n \times r_{1}}, \\
R_{U}=W_{U} V_{U}^{\top} & \text { with } & W_{U} \in \mathbb{R}^{n \times r_{2}}, V_{U} \in \mathbb{R}^{n_{t} \times r_{2}}, \\
R_{P}=W_{P} V_{P}^{\top} & \text { with } & W_{P} \in \mathbb{R}^{n \times r_{3}}, V_{P} \in \mathbb{R}^{n_{t} \times r_{3}},
\end{array}
$$

where $r_{i} \ll n$. If the residual is not initially of this form, then we assume that it can be well approximated in this way. Every Krylov scheme now performs matrix-vector multiplications of $\mathcal{A}$ and the initial residual given in (26). In [83] it was shown that the matrix-vector product with the matrix (14) can maintain the low-rank structure, only requiring an additional truncation step that can be performed cheaply compared to the cost of the full method. The construction of such a truncation function $\mathcal{T}_{\varepsilon}$ for the matrix case is further described in [46, 83].

\section{Tensor case}

The previously discussed matrix setup is of course a special case of the more general tensor problem. As we noted, algebraic operations (matrix, scalar products and additions) and the SVD re-compression procedure in the TT format allow to rewrite any classical iterative method, keeping all vectors in the tensor format and performing only structured operations [64, 4, 21, 46]. Let us denote the compression (or truncation) procedure from a vector $y$ to a vector $\widetilde{y} \approx y$ as

$$
\widetilde{y}=\mathcal{T}_{\varepsilon}(y),
$$

where by $\varepsilon$ we denote the relative truncation accuracy in the Frobenius norm. In particular, the TT-MinRes algorithm can be written as shown in Algorithm 1 .

Again the convergence behavior of the MinRES algorithm depends on the system parameters and, in order to achieve robust convergence, we need to construct a suitable preconditioner. 


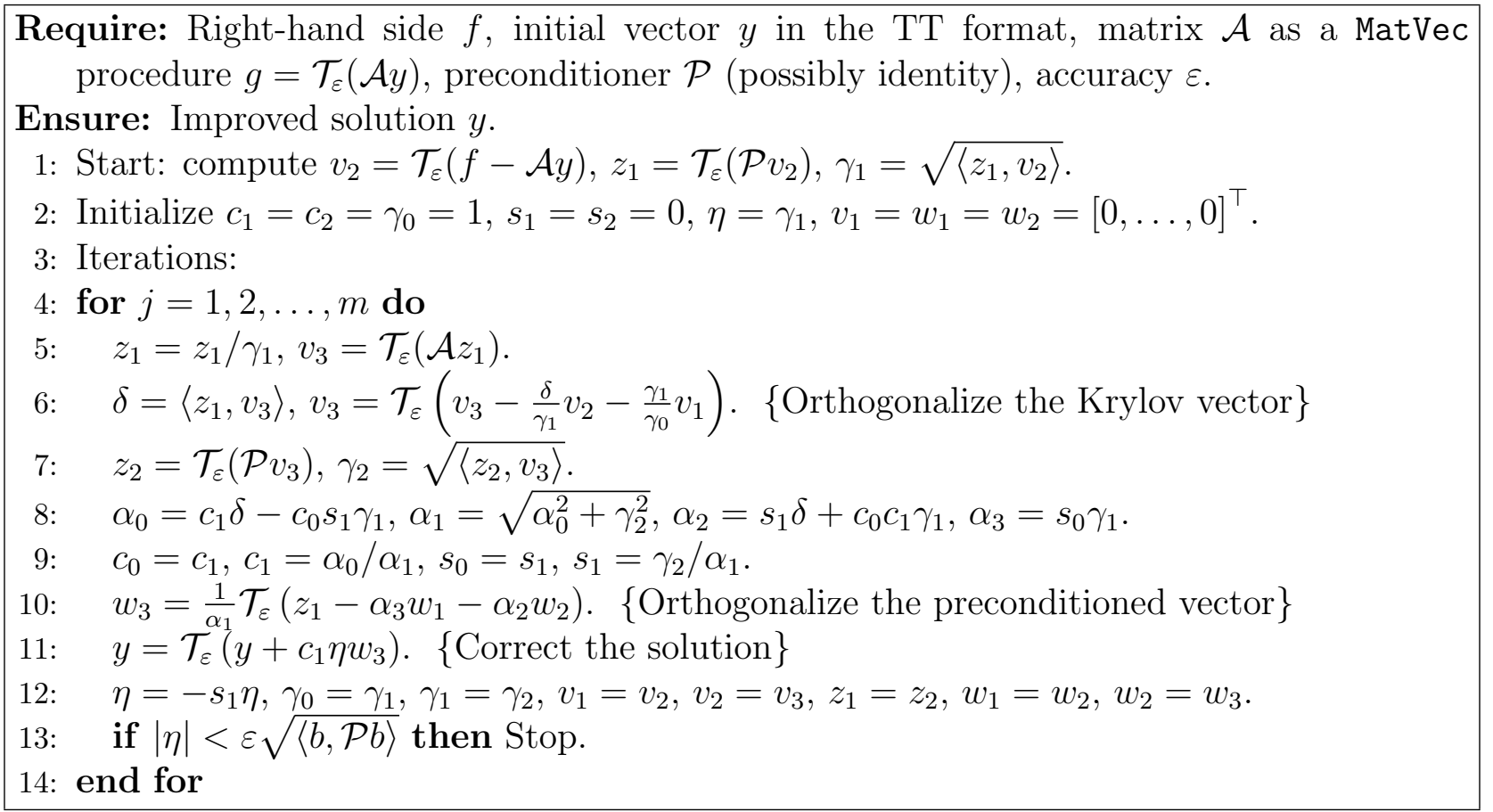

Algorithm 1: TT-MINRES

\section{Preconditioning}

We have now established the use of a low-rank or tensor Krylov method and have previously discussed block-preconditioners. It is now crucial to evaluate the preconditioners $\mathcal{P}$ given by the block-diagonal matrix/tensor in Section 3.1.

In the matrix case, the inverse of $\widetilde{S}$ from $(16)$ can be approximated by employing low-rank methods for Sylvester equations to approximately solve for $\widetilde{A}$ and $\widetilde{A}^{\top}$. Note that both of these operations mean approximately solving Sylvester-type equations, and computing one matrix multiplication.

The solution of these matrix equations is of crucial importance in many areas of science and engineering. While direct methods such as the Bartels-Stewart algorithm [29] are suitable for moderate matrix sizes, larger problems require the use of iterative schemes. Additionally the storage demand for the solution matrix is often vast, and hence low-rank methods have become a standard tool to approximate the solution of Sylvester equations [81]. The efficient use of alternating direction implicit (ADI) methods was established over the last decade [5, 8, 6, 82. The ADI scheme often gives very good approximations but requires a set of shift parameters to guarantee convergence, which can be difficult to obtain. Hackbusch and Grasedyck suggest the use of a multigrid scheme [31] that maintains the low-rank nature of the solution throughout the iteration. More reliable is the Krylov-plus-inverted-Krylov (KPIK) method, first developed in [24, 80] for Lyapunov equations and later adapted for the case of Sylvester equations [81]. This method approximates the solution of the Sylvester equation in an extended Krylov subspace that involves two sequences with each of the system matrices $C_{\alpha}$ and $\frac{1}{\sqrt{\gamma}} I_{n}-L$ in $\widetilde{A}$. Additionally, the sequence requires the inverse or approximate inverse of both $C_{\alpha}$ and $\frac{1}{\sqrt{\gamma}} I_{n}-L$ from (8) and (9). Since they are Toeplitz matrices, we can employ fast iterative solvers using circulant preconditioners, following [13].

In the tensor case, for the matrix $\widetilde{A}$, as well as for Schur complement approaches in Section 3 , we require an efficient tensor product solver, which is discussed next. 


\subsection{Alternating solvers}

It is often observed that Krylov vectors may require much larger TT ranks than the solution of the problem, see e.g. [23]. Alternating iterative tensor algorithms avoid this problem by seeking directly the elements of the tensor format for the solution. The Alternating Least Squares method (see [45] and references there) was developed to fit given data to a low-rank model. The Density Matrix Renormalization Group (DMRG) algorithm [89] was initially proposed for solution of ground state eigenvalue problems in quantum physics, and then extended to linear systems [63, 36, 38].

Given a TT format (24) for the approximate solution $y$, we may collect its first, resp. last, $k$ TT factors into the interface matrices,

$$
\begin{gathered}
Y^{(1: k)}\left(\overline{i_{1} \ldots i_{k}}, \alpha_{k}\right)=\sum_{\alpha_{1}, \ldots, \alpha_{k-1}=1}^{r_{1}, \ldots, r_{k-1}} \mathbf{y}_{\alpha_{1}}^{(1)}\left(i_{1}\right) \mathbf{y}_{\alpha_{1}, \alpha_{2}}^{(2)}\left(i_{2}\right) \cdots \mathbf{y}_{\alpha_{k-1}, \alpha_{k}}^{(k)}\left(i_{k}\right), \quad Y^{(1: k)} \in \mathbb{R}^{n_{1} \cdots n_{k} \times r_{k}}, \\
Y^{(k: d)}\left(\alpha_{k-1}, \overline{i_{k} \ldots i_{d}}\right)=\sum_{\alpha_{k}, \ldots, \alpha_{d-1}=1}^{r_{d-1}} \mathbf{y}_{\alpha_{k-1}, \alpha_{k}}^{(k)}\left(i_{k}\right) \cdots \mathbf{y}_{\alpha_{d-1}}^{(d)}\left(i_{d}\right), \quad Y^{(k: d)} \in \mathbb{R}^{r_{k-1} \times n_{k} \cdots n_{d}} .
\end{gathered}
$$

Then we assemble the frame matrix,

$$
Y_{\neq k}=Y^{(1: k-1)} \otimes I_{n_{k}} \otimes\left(Y^{(k+1: d)}\right)^{\top} \in \mathbb{R}^{n_{1} \cdots n_{d} \times r_{k-1} n_{k} r_{k}}
$$

which includes all TT factors but $\mathbf{y}^{(k)}$, hence the notation. Note that the column size of the frame matrix is exactly equal to the number of elements in the TT block $\mathbf{y}^{(k)}$. The TT format (24) can be seen as a linear map, induced by the frame matrix, i.e. $y=Y_{\neq k} y^{(k)}$, where we denote by $y^{(k)}$ the vector of elements of the $k$-th TT block, $y^{(k)}\left(\overline{\alpha_{k-1} i_{k} \alpha_{k}}\right)=\mathbf{y}_{\alpha_{k-1}, \alpha_{k}}^{(k)}\left(i_{k}\right)$.

Now let us consider $A y=f$ as an overdetermined linear linear system on $y^{(k)}$, provided the TT format (24) is inserted instead of $y$, i.e. $A Y_{\neq k} y^{(k)}=f$. A simple way to solve this system is to project it onto the frame matrix. We solve

$$
A_{k} y^{(k)}=f_{k}, \quad A_{k}=Y_{\neq k}^{\top} A Y_{\neq k}, \quad f_{k}=Y_{\neq k}^{\top} f
$$

Using the orthogonalizations of the TT blocks via the QR decompositions of their matrix reshapings, we can always make the frame matrix orthogonal [79], that is $Y_{\neq k}^{\top} Y_{\neq k}=I$. This ensures the stability of the problem, $\operatorname{cond}\left(A_{k}\right) \leq \operatorname{cond}(A)$ if $A+A^{\top}>0$. Iterating for all dimensions $k=1, \ldots, d$ (hence the name "alternating"), we obtain the simple one-block DMRG, or Alternating Linear Scheme (ALS) [36] algorithm.

However, in this scheme all frame matrices, and hence all TT blocks, have fixed sizes, prescribed by the TT ranks of the initial guess. This is very inconvenient: in most cases it is difficult to predict TT ranks of the solution for a given accuracy, and we would like to determine them adaptively. Another problem is the local convergence of the ALS method [76]: the result is highly dependent on the initial guess and may give an unsatisfactory approximation.

There are two principal ways to solve the first (technical) problem of the TT rank adaptivity during the iterative process. Note that the main issue is how to increase the ranks; to decrease them, it is sufficient to perform the SVD re-compression procedure, as pointed out in Section 4.1. One way to increase the ranks is given by the DMRG algorithm in its initial, 
two-block version [89]. Instead of one block $y^{(k)}$, we update both $y^{(k)}$ and $y^{(k+1)}$ at each step and adapt the TT rank $r_{k}$. However, for linear systems with non-symmetric matrices, even the two-block DMRG method may converge to an incorrect solution [23]. A better way to increase TT ranks is to enrich TT blocks explicitly. On step $1 \leq k \leq d$, after the solution of (29), we may expand

$$
\mathbf{y}^{(k)}\left(i_{k}\right):=\left[\begin{array}{ll}
\mathbf{y}^{(k)}\left(i_{k}\right) & \mathbf{z}_{k}^{(k)}\left(i_{k}\right)
\end{array}\right], \quad \mathbf{y}^{(k+1)}\left(i_{k+1}\right):=\left[\begin{array}{c}
\mathbf{y}^{(k+1)}\left(i_{k+1}\right) \\
\mathbf{0}
\end{array}\right],
$$

where $\mathbf{z}_{k}^{(k)} \in \mathbb{R}^{r_{k-1} \times n_{k} \times \rho_{k}}$ is some auxiliary tensor, and the zero-block in $\mathbf{y}^{(k+1)}$ has the corresponding sizes $\rho_{k} \times n_{k+1} \times r_{k+1}$. This step does not impact the whole solution $y$. However, when we proceed to the next block $\mathbf{y}^{(k+1)}$, the interface $Y^{(1: k)}$ and the frame $Y_{\neq k+1}$ matrices carry $\mathbf{z}_{k}^{(k)}$, so the Galerkin reduction 29$)$ in the step $k+1$ is performed to a wider basis than in the ALS method. This can not only increase the TT rank, but also facilitate the convergence, if we select the augmentation $\mathbf{z}_{k}^{(k)}$ properly.

The idea of the AMEn algorithm proposed in [23] is to combine the alternating iteration with the steepest descent method. The latter updates the solution by adding the scaled residual, $y:=y+z h$, where $z \approx f-A y$ and $h$ is a scalar weight. If $y$ and $z$ are defined by their TT formats, the summation is computed as

$$
y\left(\overline{i_{1} \ldots i_{d}}\right)+h z\left(\overline{i_{1} \ldots i_{d}}\right)=\left[\begin{array}{ll}
\mathbf{y}^{(1)}\left(i_{1}\right) & \mathbf{z}^{(1)}\left(i_{1}\right)
\end{array}\right]\left[\begin{array}{ll}
\mathbf{y}^{(2)}\left(i_{2}\right) & \\
& \mathbf{z}^{(2)}\left(i_{2}\right)
\end{array}\right] \cdots\left[\begin{array}{c}
\mathbf{y}^{(d)}\left(i_{d}\right) \\
h \mathbf{z}^{(d)}\left(i_{d}\right)
\end{array}\right] .
$$

Note that the first factor here has the same form as the enrichment of $\mathbf{y}^{(k)}$ in $(30)$; therefore, (30) performs the first step of the TT format addition. This is sufficient, since in the next step we solve (29) and recover correct $z$-related entries in $\mathbf{y}^{(k+1)}$ that minimize the energy function if $A=A^{\top}>0$. Due to this property, the new method was called AMEn (alternating minimal energy).

To compute $\mathbf{z}_{k}^{(k)}$ in practice, it is sufficient to provide a very rough approximation of the residual. We prepare some initial guess for $z$ in the TT format with ranks $\rho_{1}, \ldots, \rho_{d-1}$ and update it towards $f-A y$ by the secondary ALS iteration, minimizing the distance $\|z-(f-A y)\|_{2}$. The entire procedure is summarized in Algorithm 2. Notice that after the enrichment (30) we need to orthogonalize $\mathbf{y}^{(k)}$ explicitly in order to make the frame matrices orthogonal.

In most cases, the enrichment ranks $\rho_{k} \lesssim \rho \lesssim 10$ are sufficient. To make the computational complexity in Lines $4,5,7$ independent of $d$, we reuse some intermediate data during the subsequent iteration $k=1, \ldots, d$ (see [22, 63, 23] for details).

At this juncture we wish to remark on the utility of the approach presented when nonlinear problems, such as the previously mentioned phase-field equations, are considered. Our crucial contribution in this work is the introduction of low-rank solvers for the saddle point problems that arise for the linear problems. For nonlinear problems one typically applies an outer solver such as a Picard-type or Newton-type method [57]. At the heart of these methods, and indeed almost all nonlinear solution strategies, lies the solution of a large-scale system in saddle point form for which the low-rank strategies we have described can be applied. In future work we wish to carry out a thorough analysis of this setting: from our experience with low-rank methods for classical derivative and nonlinear equations the structure of the saddle point system (14) will be similar, but the particular blocks, e.g. the FDE block $A$, can consist of a sum of tensors due it being the linearization of a nonlinear low-rank operator. 


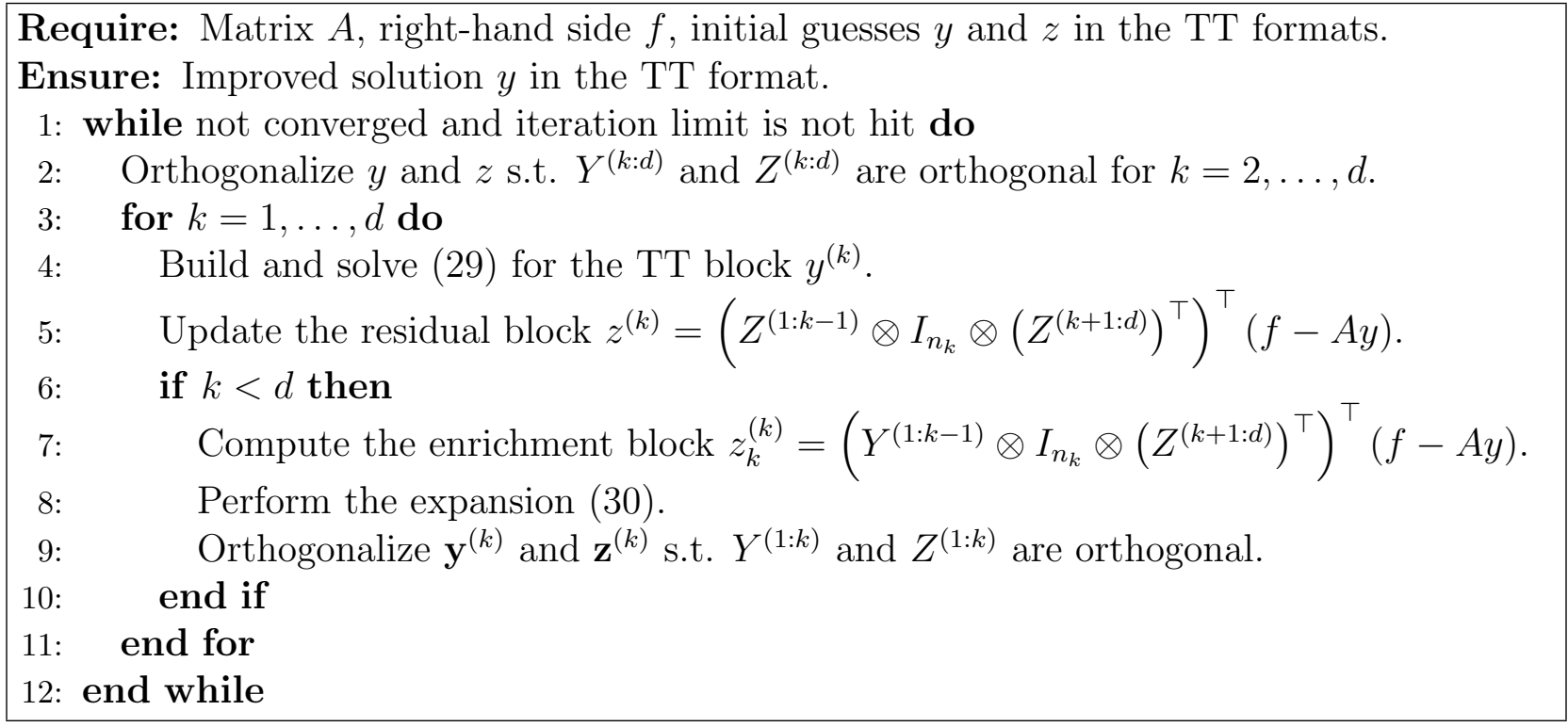

Algorithm 2: AMEn method

\section{Numerical results}

We solve the problem (14) in the QTT format for different inputs and parameters, and compare three procedures:

"SC1" the First Schur Complement scheme (18), where the AMEn Algorithm 2 is applied to solve linear systems;

"SC2" the Second Schur Complement scheme (20), again with the AMEn solver; and "MR" the MinRES Algorithm 1 with the preconditioner (16).

In the MR approach, the linear systems with the matrices $\widetilde{A}$ and $\widetilde{A}^{\top}$ are also solved using the AMEn method. The three components $y, u$ and $p$ and the blocks in the matrix (14) are kept in separate TT formats. Since the matrix $A$ has considerable TT ranks (up to 20), it is not efficient to multiply it by vectors exactly and then perform the SVD truncation. Instead, we approximate the matrix-by-vector product iteratively using a special variant of Algorithm 2. where $A=I$ and $f=\widetilde{A} y$. Further implementation details are given in the Appendix.

As a widely used error indicator, we consider the relative discrepancy of the state vector $y$ in the Frobenius norm. Given some reference vector $y_{\star}$, we denote

$$
\mathcal{E}\left(y_{\star}\right)=\frac{\left\|y-y_{\star}\right\|_{2}}{\left\|y_{\star}\right\|_{2}}
$$

The examples $2^{2}$ were implemented using the TT-Toolbox packag $\AA^{3}$ and conducted on one core of the MPI otto cluster, an Intel Xeon X5650 CPU at 2.67GHz, in Matlab R2012a.

\subsection{Complete data, two-dimensional space}

In the first test both observation and control are defined on the whole domain $[0,1]^{2}$. Therefore, $M_{1}$ and $M_{3}$ are identity matrices, and both Schur complements are well defined. We investigate their performance and compare with the TT-MinRES approach.

${ }^{2}$ The source codes are available at http://www .mpi-magdeburg.mpg.de/2866211/supplementaries.zip 3 http://github.org/oseledets/TT-Toolbox, version Nov 10, 2014 
Table 1: Complete data test. Left: CPU times (sec.), maximal TT ranks and iterations vs. grid level $l$. Right: discrepancies between solutions computed by SC2, Minres, Full and SC1 schemes.

\begin{tabular}{c|cc|cc|ccc|c||ccc}
$l$ & \multicolumn{2}{|c|}{ SC1 } & \multicolumn{2}{c|}{ SC2 } & \multicolumn{3}{c|}{ MR } & Full & SC2 & MR & Full \\
\hline & time & rank & time & rank & time & rank & iter & time & & $\mathcal{E}\left(y_{S C 1}\right)$ & \\
3 & 0.596 & 10 & 1.396 & 9 & 2.980 & 13 & 9 & 0.552 & $1.47 \mathrm{e}-6$ & $2.24 \mathrm{e}-7$ & $2.51 \mathrm{e}-7$ \\
4 & 0.489 & 12 & 1.532 & 14 & 4.823 & 20 & 11 & 7.695 & $2.45 \mathrm{e}-6$ & $3.11 \mathrm{e}-7$ & $2.90 \mathrm{e}-7$ \\
5 & 1.021 & 18 & 4.103 & 21 & 11.45 & 26 & 15 & 232.3 & $7.07 \mathrm{e}-6$ & $5.97 \mathrm{e}-7$ & $5.64 \mathrm{e}-7$ \\
6 & 2.280 & 22 & 11.69 & 27 & 24.70 & 36 & 15 & 7513 & $5.06 \mathrm{e}-5$ & $8.63 \mathrm{e}-7$ & $8.36 \mathrm{e}-7$ \\
7 & 5.061 & 25 & 27.61 & 36 & 83.99 & 49 & 15 & - & $2.00 \mathrm{e}-4$ & $9.14 \mathrm{e}-7$ & - \\
8 & 10.46 & 28 & 55.45 & 41 & 245.7 & 62 & 15 & - & $8.16 \mathrm{e}-4$ & $1.18 \mathrm{e}-6$ & - \\
9 & 18.90 & 29 & 99.94 & 45 & 984.9 & 78 & 17 & - & $2.41 \mathrm{e}-3$ & $2.04 \mathrm{e}-6$ & - \\
10 & 28.03 & 29 & 175.1 & 51 & 3494 & 100 & 17 & - & $1.03 \mathrm{e}-2$ & $5.27 \mathrm{e}-6$ & - \\
11 & 47.49 & 29 & 257.8 & 55 & 33005 & 255 & 25 & - & $2.18 \mathrm{e}-2$ & $2.52 \mathrm{e}-5$ & -
\end{tabular}

The constraint problem is the equation $(1 \mathrm{~b})$ in 2 spatial variables plus time, with $\alpha=0.5$, $\beta_{1}=1.5, \beta_{2}=1.5$ and $f=0$. The level-l discretization grid contains $2^{l} \times 2^{l} \times 2^{2 l}$ points, lying uniformly in space and time, i.e.,

$$
\begin{aligned}
& x_{1}(i)=i h, \quad x_{2}(j)=j h, \quad t(k)=k \tau, \\
& i, j=1, \ldots, 2^{l}, \quad k=1, \ldots, 2^{2 l}, \quad h=\frac{1}{2^{l}+1}, \quad \tau=h^{2} .
\end{aligned}
$$

The target function is

$$
\bar{y}\left(x_{1}, x_{2}, t\right)=10 \cos \left(10 x_{1}\right) \sin \left(x_{1} x_{2}\right) .
$$

Boundary and initial conditions are taken to be homogeneous. We vary the grid level $l$, the regularization parameter $\gamma$ and the tensor approximation tolerance $\varepsilon$. We use the same threshold $\varepsilon$ both for the tensor approximation and also as the stopping tolerance for our numerical schemes.

Performance with respect to the grid size

We fix $\gamma=10^{-6}, \varepsilon=10^{-6}$, and vary the grid size in the range $l=3, \ldots, 11$. The results are shown in Table 1. We can see that the iteration numbers for MinRES are quite robust with respect to the varying mesh-sizes. Nevertheless, the rank increase with each refinement slows the method down significantly. The SC2 approach also suffers from a rank increase but less significantly than the MinREs. As this is the most benign case with full observation and control domain, the SC1 method performs outstandingly with almost no increase in the ranks for the final refinements. We further compare the solutions of the three different approaches and can see that the MiNRES and SC1 approach show the best coincidence.

To demonstrate the importance of the QTT compression, we also compare our approaches with the "Full" scheme, where (14) is solved via classical MinRES with the full storage of all vectors. Toeplitz matrices were multiplied by vectors via the FFT, and the linear systems with $\widetilde{A}$ and $\widetilde{A}^{\top}$ from $(16)$ were solved using the BICGSTAB method. The solution accuracy is good, but the CPU time grows dramatically and makes the calculations prohibitively expensive for $l>6$.

Performance with respect to the regularization parameter (Table 2)

In the second experiment we examine the behavior of the methods with respect to the regularization parameter $\gamma$. For an application the choice of regularization parameter is often 
Table 2: Complete data test. Left: CPU times (sec.) and discrepancies with the observation data. Right: discrepancies with SC1.

\begin{tabular}{|c|c|c|c|c|c|c|c|c|}
\hline$\gamma$ & \multicolumn{2}{|c|}{ SC1 } & \multicolumn{2}{|c|}{$\mathrm{SC} 2$} & \multicolumn{2}{|c|}{ MR } & $\mathrm{SC} 2$ & MR \\
\hline & time & $\mathcal{E}(\bar{y})$ & time & $\mathcal{E}(\bar{y})$ & time & $\mathcal{E}(\bar{y})$ & $\mathcal{E}\left(y_{S C 1}\right)$ & $\mathcal{E}\left(y_{S C 1}\right)$ \\
\hline $10^{-2}$ & 23.91 & $9.33 \mathrm{e}-1$ & 31.56 & $9.33 \mathrm{e}-1$ & 4884 & $9.33 \mathrm{e}-1$ & $4.53 \mathrm{e}-5$ & $4.12 \mathrm{e}-5$ \\
\hline $10^{-4}$ & 14.47 & $3.31 \mathrm{e}-1$ & 26.97 & $3.31 \mathrm{e}-1$ & 707.0 & $3.31 \mathrm{e}-1$ & $1.39 \mathrm{e}-4$ & $6.94 \mathrm{e}-6$ \\
\hline $10^{-6}$ & 5.226 & $9.53 \mathrm{e}-2$ & 27.72 & $9.53 \mathrm{e}-2$ & 88.38 & $9.53 \mathrm{e}-2$ & $1.76 \mathrm{e}-4$ & $9.51 \mathrm{e}-7$ \\
\hline $10^{-8}$ & 1.354 & $5.27 \mathrm{e}-3$ & 29.51 & $5.29 \mathrm{e}-3$ & 17.18 & $5.27 \mathrm{e}-3$ & $4.23 \mathrm{e}-4$ & $1.18 \mathrm{e}-6$ \\
\hline $10^{-10}$ & 1.217 & $5.75 \mathrm{e}-5$ & 31.26 & $4.28 \mathrm{e}-4$ & 5.531 & $5.75 \mathrm{e}-5$ & $4.24 \mathrm{e}-4$ & $9.91 \mathrm{e}-7$ \\
\hline $10^{-12}$ & 1.189 & $1.57 \mathrm{e}-6$ & 29.43 & $3.78 \mathrm{e}-4$ & 2.450 & $5.75 \mathrm{e}-7$ & $3.78 \mathrm{e}-4$ & $1.48 \mathrm{e}-6$ \\
\hline
\end{tabular}

Table 3: Complete data test. Left: CPU times (sec.) of three methods vs. tensor approximation tolerance $\varepsilon$. Right: discrepancies of SC2 and MinRES with SC1.

\begin{tabular}{c|ccc||cc}
$\varepsilon$ & SC1 & SC2 & MR & $\frac{\left\|y_{S C 2}-y_{S C 1}\right\|}{\left\|y_{S C 1}\right\|}$ & $\frac{\left\|y_{M R}-y_{S C 1}\right\|}{\left\|y_{S C 1}\right\|}$ \\
\hline $10^{-2}$ & 0.728 & 1.471 & 3.400 & $2.50 \mathrm{e}-1$ & $7.06 \mathrm{e}-3$ \\
$10^{-4}$ & 1.449 & 4.899 & 9.869 & $1.38 \mathrm{e}-2$ & $1.01 \mathrm{e}-4$ \\
$10^{-6}$ & 4.962 & 27.53 & 75.07 & $1.99 \mathrm{e}-4$ & $9.11 \mathrm{e}-7$ \\
$10^{-8}$ & 17.26 & 163.6 & 504.1 & $1.18 \mathrm{e}-6$ & $8.57 \mathrm{e}-9$ \\
$10^{-10}$ & 37.34 & 880.2 & 2846 & $3.76 \mathrm{e}-9$ & $6.27 \mathrm{e}-10$
\end{tabular}

determined by techniques such as $L$-curve analysis [32] and discrepancy principle [26]. As we do not focus on a particular application but rather a methodological development we want to illustrate the robustness of our method regarding for a wide range of regularization parameters. In our experiments we use the grid level is $l=7$, and $\varepsilon=10^{-6}$. According to Table 2, we can see that the computed state approaches the desired state further when $\gamma$ gets smaller. Due to the Dirichlet boundary conditions, we neither observe nor control the boundary, and consider only the inner grid points in the discrete model. The error indicator $\mathcal{E}(\bar{y})$ involves only the inner points as well. Therefore, it is possible to approach $\bar{y}$ when $\gamma \rightarrow 0$. As a by-product, the CPU times of all methods decrease with $\gamma$. Since the matrices $M_{1}, M_{2}$ and $M_{3}$ are scaled identities, the Schur complement (17) becomes $S=\frac{1}{\tau h^{2}} A A^{\top}+\frac{\tau h^{2}}{\gamma} I$, which tends to an identity when $\gamma \rightarrow 0$, and becomes easier to invert. This is clearly reflected by the SC1 and MR methods. However, the $\mathrm{SC} 2$ is slower: its bottleneck is the inversion of $A$, which is performed independently of $\gamma$.

Performance with respect to the accuracy threshold (Table 3)

Finally, we fix $l=7, \gamma=10^{-6}$, and vary $\varepsilon$ from $10^{-1}$ to $10^{-10}$; we show the results in Table 3. It is not surprising that all methods require more computing time to obtain the desired accuracy. The discrepancies demonstrate almost perfect linear dependence on $\varepsilon$.

\subsection{Incomplete data, two-dimensional space}

We now investigate the following more challenging scenario, reducing the observation and control domains to $\Omega_{\bar{y}}=\Omega_{u}=\left[\frac{3}{8}, \frac{5}{8}\right]^{2}$. Consequently, the first Schur Complement scheme is not applicable, and we test the second approach. Other parameters are the same as in the previous example. 
Table 4: Incomplete data test. Left: CPU times (sec.), maximal TT ranks and iterations vs. grid level $l$. Right: solution errors.

\begin{tabular}{c|cc|ccc|c||cc|c|c}
$l$ & \multicolumn{2}{|c|}{ SC2 } & \multicolumn{3}{|c|}{ MR } & Full & \multicolumn{2}{c|}{ SC2 } & MR & Full \\
\hline & time & rank & time & rank & iter & time & $\mathcal{E}\left(y_{\star}\right)$ & $\mathcal{E}(\bar{y})$ & $\mathcal{E}\left(y_{\star}\right)$ & $\mathcal{E}\left(y_{\star}\right)$ \\
3 & 1.081 & 23 & 5.247 & 44 & 11 & 5.521 & $4.25 \mathrm{e}-7$ & $2.42 \mathrm{e}-3$ & $2.27 \mathrm{e}-4$ & $8.31 \mathrm{e}-9$ \\
4 & 2.426 & 26 & 27.88 & 138 & 15 & 124.1 & $1.69 \mathrm{e}-6$ & $9.12 \mathrm{e}-3$ & $1.49 \mathrm{e}-4$ & $8.93 \mathrm{e}-9$ \\
5 & 7.039 & 42 & 1018 & 752 & 17 & 3542 & $3.34 \mathrm{e}-6$ & $1.82 \mathrm{e}-2$ & $3.68 \mathrm{e}-4$ & $3.53 \mathrm{e}-5$ \\
6 & 20.96 & 56 & & - & & - & $1.33 \mathrm{e}-5$ & $2.98 \mathrm{e}-2$ & - & - \\
7 & 54.19 & 64 & & - & & - & $4.29 \mathrm{e}-5$ & $3.62 \mathrm{e}-2$ & - & - \\
8 & 120.0 & 72 & & - & & - & $3.21 \mathrm{e}-4$ & $3.76 \mathrm{e}-2$ & - & - \\
9 & 223.7 & 75 & & - & & - & $1.28 \mathrm{e}-3$ & $3.76 \mathrm{e}-2$ & - & - \\
10 & 414.4 & 76 & & - & & - & $4.25 \mathrm{e}-3$ & $3.78 \mathrm{e}-2$ & - & - \\
11 & 743.7 & 81 & & - & & - & $1.05 \mathrm{e}-2$ & $3.94 \mathrm{e}-2$ & - & -
\end{tabular}

Performance with respect to the grid size (Table 4)

The first parameter that we vary is the grid level $l$. The regularization parameter is $\gamma=10^{-6}$, the approximation tolerance $\varepsilon=10^{-6}$, and the fractional orders are $\alpha=0.5$, $\beta_{1}=\beta_{2}=1.5$. The results are shown in Table 4.

In this example, the number of MinRES iterations is moderate, but the TT ranks grow quickly, making the computations prohibitively expensive for grids larger than $l=5$.

To estimate the error in the solution, we compute the reference solution $y_{\star}$ using the second Schur Complement scheme with a smaller threshold $\varepsilon_{\star}=10^{-8}$. As an important indicator for the incomplete observation and control, we also demonstrate the deviation of the solution $y$ from the prescribed data $\bar{y}$ on the observation domain.

A drawback of the Schur Complement scheme which we observe is the growth of the error, by a factor 3 on average from level to level. This is because the condition number of the system matrix grows like $\left(2^{l}\right)^{\beta}=\left(2^{1} .5\right)^{l} \approx 3^{l}$, and so does the error when we compute the approximate inverse matrix in (21). To verify that the error comes from approximation of $Q$, not from other steps of the scheme, we solve (21) with a higher accuracy (say, $10^{-8}$ ), while all other steps in (20) are performed with the same $\varepsilon=10^{-6}$. For $l=9$, it gives $\frac{\left\|y_{S C 2}-y_{\star}\right\|}{\left\|y_{\star}\right\|} \approx 1.58 \times 10^{-5}$ in about 11 minutes.

\section{Performance with respect to the regularization parameter (Table 5)}

When the solution is controlled on a part of the domain, the influence of the regularization parameter $\gamma$ becomes more interesting numerically. As previously, we fix the grid level to $l=7$ and set $\varepsilon=10^{-6}$. The reference solution is computed with the threshold $\varepsilon_{\star}=10^{-8}$. We omit the experiment with MinRES, since it would require very large TT ranks.

In this test, the part $M_{1} A^{-1} M_{3}\left(\gamma M_{2}\right)^{-1} M_{3}^{\top}$ of the Schur complement matrix in (20) is indefinite. For small $\gamma$ the whole Schur complement may become indefinite, which makes the calculations difficult. Therefore, we are limited by the range of $\gamma$ we can experiment with. However, the scheme is quite reliable for $\gamma \geq 10^{-7}$. The solution error is almost stable, and the complexity grows moderately. The discrepancy is proportional to $\sqrt{\gamma}$, as expected.

Performance with respect to $\alpha$ and $\beta$ (Fig. 1)

Since the order of a fractional derivative is a continuous quantity, it is interesting to test the algorithms for a range of orders. In particular, in the first test we vary $\alpha \in[0.1,1]$ and 
Table 5: Incomplete data test. CPU times (sec.) and accuracy of the $\mathrm{SC} 2$ method vs. regularization parameter $\gamma$

\begin{tabular}{c|cc|cc}
$\gamma$ & time & rank & $\mathcal{E}\left(y_{\star}\right)$ & $\mathcal{E}(\bar{y})$ \\
\hline $10^{-1}$ & 37.66 & 44 & $1.65 \mathrm{e}-6$ & $9.95 \mathrm{e}-1$ \\
$10^{-2}$ & 41.62 & 47 & $1.01 \mathrm{e}-5$ & $9.61 \mathrm{e}-1$ \\
$10^{-4}$ & 47.02 & 52 & $3.67 \mathrm{e}-5$ & $3.48 \mathrm{e}-1$ \\
$10^{-6}$ & 54.65 & 64 & $4.40 \mathrm{e}-5$ & $3.62 \mathrm{e}-2$ \\
$10^{-7}$ & 74.23 & 68 & $6.42 \mathrm{e}-5$ & $9.66 \mathrm{e}-3$
\end{tabular}

$\beta_{1}=\beta_{2} \in[1.1,2]$. We show two-dimensional plots of computation times, TT ranks and errors in Fig 1 .

The TT ranks and CPU times grow towards lines $\alpha=1$ and $\beta=1$. To explain this, recall that the matrix $C_{\alpha}$ approaches identity for $\alpha \rightarrow 0$ and turns into the standard first-order difference at $\alpha=1$, and the condition number cond $\left(C_{\alpha}\right)$ grows monotonously for $\alpha \in(0,1]$. However, the Riemann-Liouville matrix $L_{\beta}$ behaves differently: at $\beta=1$, the matrix is equal to the scaled second-order difference, $L_{1}=\frac{h}{2} L_{2}$. When $\beta>1$, the matrix $L_{\beta}$ depends continuously on $\beta$, so the condition number should have an extremum on $[1,2]$, which is apparently a minimum near $\beta=1.2$. Large conditioning makes the problem harder to solve when $\beta$ approaches 1. Even more difficult are the cases $\beta<1$ and $\alpha>1$ : the matrices $C_{\alpha}$ and $L_{\beta}$ become indefinite, and the tensor product solver struggles.

Nevertheless, for parabolic cases $\alpha \in(0,1]$ and $\beta \in(1,2]$, our scheme is fairly robust. The bottom left plot in Fig. 1 shows that the relative error sits at the level $10^{-4}-10^{-5}$ for the entire range of orders of differentiation. The deviation from the target vector (Fig. 1 , bottom right) is also reasonable: the closer the orders are to zero, the closer the operators are to identities, and hence they impose a less severe constraint to the optimization of the distance $\|y-\bar{y}\|$.

Performance with respect to $\beta_{1}$ and $\beta_{2}$ (Fig. 2)

We now fix $\alpha=0.5$, and vary $\beta_{1}, \beta_{2}$ within the range $[1.1,2]$. The layout of Fig. 2 is the same as in Fig. 1, only the CPU times are now presented without the logarithmic scale, since they do not vary as strongly. Again, both CPU times and TT ranks increase towards 1. As opposed to the picture w.r.t. $\alpha$ and $\beta$, there is an anisotropy w.r.t. $\beta_{1}$ and $\beta_{2}$, since the target function $\bar{y}\left(x_{1}, x_{2}\right)$ is not symmetric w.r.t. $x_{1}$ and $x_{2}$. Both error indicators behave similarly to those in the previous figure.

Experiment with variable coefficients (Table 6)

A more general FDE involves variable coefficients. In particular, a two-dimensional example from [87] reads

$$
\left({ }_{0}^{\mathrm{C}} D_{t}^{\alpha}-p_{+}{ }_{0}^{\mathrm{RL}} D_{x_{1}}^{\beta_{1}}-p_{-}{ }_{x_{1}}^{\mathrm{RL}} D_{1}^{\beta_{1}}-q_{+}{ }_{0}^{\mathrm{RL}} D_{x_{2}}^{\beta_{2}}-q_{-}{ }_{x_{2}}^{\mathrm{RL}} D_{1}^{\beta_{2}}\right) y\left(x_{1}, x_{2}, t\right)+u\left(x_{1}, x_{2}, t\right)=0,
$$

where $p_{+}=\Gamma(1.2) x_{1}^{\beta_{1}}, p_{-}=\Gamma(1.2)\left(2-x_{1}\right)^{\beta_{1}}, q_{+}=\Gamma(1.2) x_{2}^{\beta_{2}}, q_{-}=\Gamma(1.2)\left(2-x_{2}\right)^{\beta_{2}}$ are the coefficient functions, and the orders are $\alpha=0.5, \beta_{1}=\beta_{2}=1.8$. Notice that the left and right Riemann-Louiville derivatives enter the equation with different coefficients. After the Grünwald-Letnikov discretization, the matrix $A$ reads

$$
A=C_{\alpha} \otimes I_{n_{1} n_{2}}-I_{n_{t}} \otimes\left(P_{+} T_{\beta_{1}}+P_{-} T_{\beta_{1}}^{\top}\right) \otimes I_{n_{2}}-I_{n_{t}} \otimes I_{n_{1}} \otimes\left(Q_{+} T_{\beta_{2}}+Q_{-} T_{\beta_{2}}^{\top}\right)
$$


Figure 1: Incomplete data test. CPU times, TT ranks and errors vs. $\alpha$ ( $x$ axis) and $\beta$ ( $y$ axis).

$\log _{10}(\mathrm{CPU}$ time, sec.)
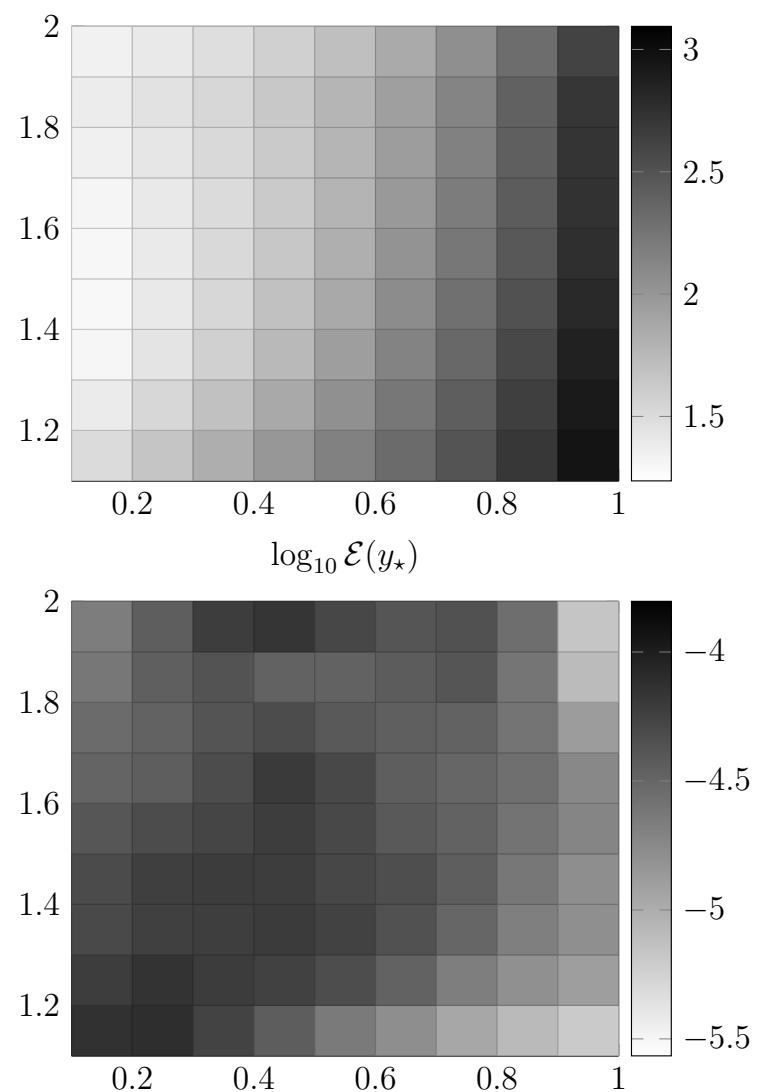

maximal TT rank
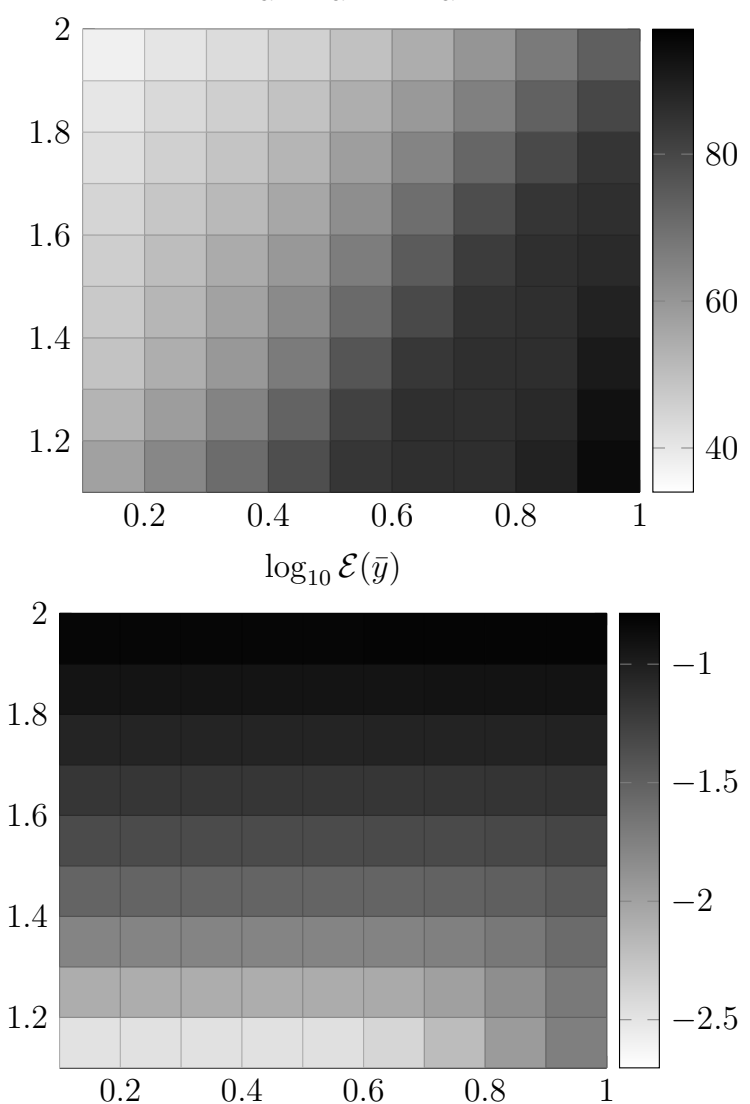

where $T_{\beta}$ is from (6), $P_{+}, P_{-}, Q_{+}, Q_{-}$are diagonal matrices with the grid values of $p_{+}, p_{-}, q_{+}, q_{-}$, respectively. We also consider a time-dependent desired state

$$
\bar{y}\left(x_{1}, x_{2}, t\right)=10 \cos \left(10 x_{1}\right) \sin \left(x_{1} x_{2}\right)(1-\exp (-5 t)) .
$$

All other parameters are the same as in the previous test.

The results are shown in Table 6. Since the coefficient admits a low-rank structure, the behavior of the methods is qualitatively the same as in the constant coefficient case. The CPU times are larger due to larger condition numbers of the matrices involved.

\subsection{Three-dimensional problem, incomplete data}

To verify the applicability of our technique to a significantly larger problem, we solve the problem $1 \mathrm{cc}$ in a three-dimensional space domain plus time. We set $\Omega=[0,1]^{3}, \alpha=0.5$, $\beta_{1}=\beta_{2}=\beta_{3}=1.5$, and $f=0$. The target function is given by

$$
\bar{y}\left(x_{1}, x_{2}, x_{3}, t\right)=e^{-64 r^{2}}, \quad r^{2}=\left(x_{1}-0.5\right)^{2}+\left(x_{2}-0.5\right)^{2}+\left(x_{3}-0.5\right)^{2} .
$$

Boundary and initial conditions are zeros. On discretization level $l$ we use the uniform $2^{l} \times 2^{l} \times 2^{l} \times 2^{2 l}$ grid. The observation and control domains are $\Omega_{\bar{y}}=\Omega_{u}=\left[\frac{3}{8}, \frac{5}{8}\right]^{3}$, which is about $1.5 \%$ of the whole volume. The regularization and stopping tolerances are set to $\gamma=\varepsilon=10^{-6}$. 
Figure 2: Incomplete data test. CPU times, TT ranks and errors vs. $\beta_{1}$ ( $x$ axis) and $\beta_{2}$ (y axis). CPU time, sec.
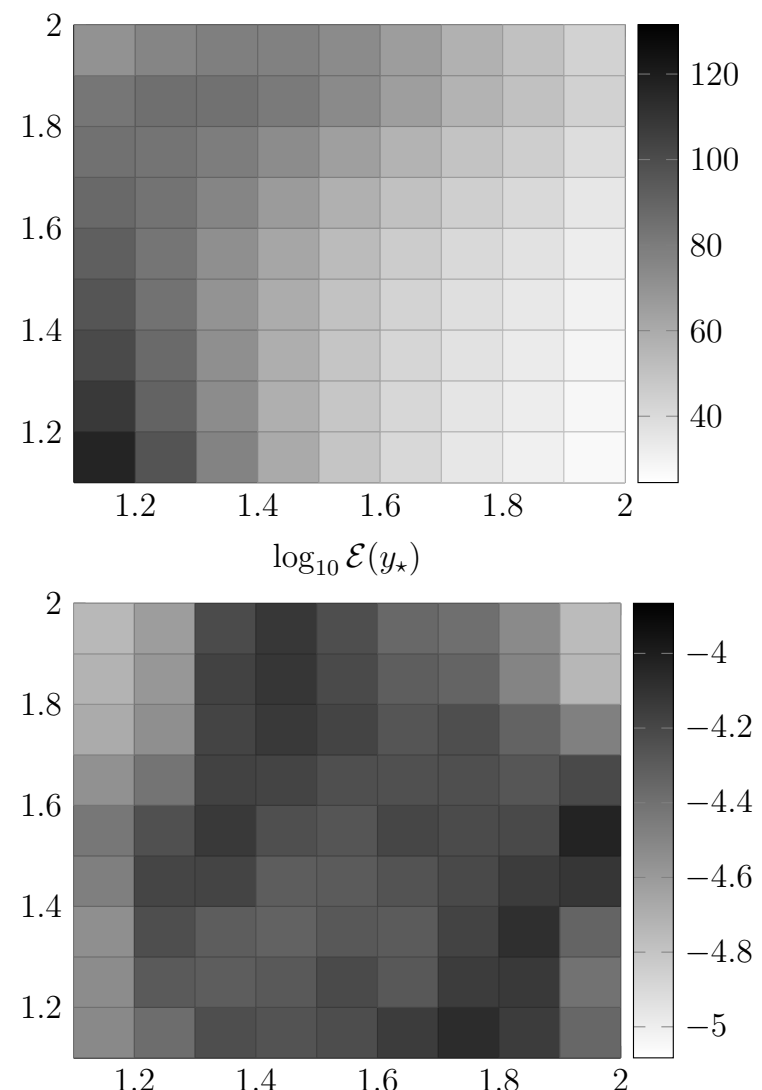

maximal TT rank
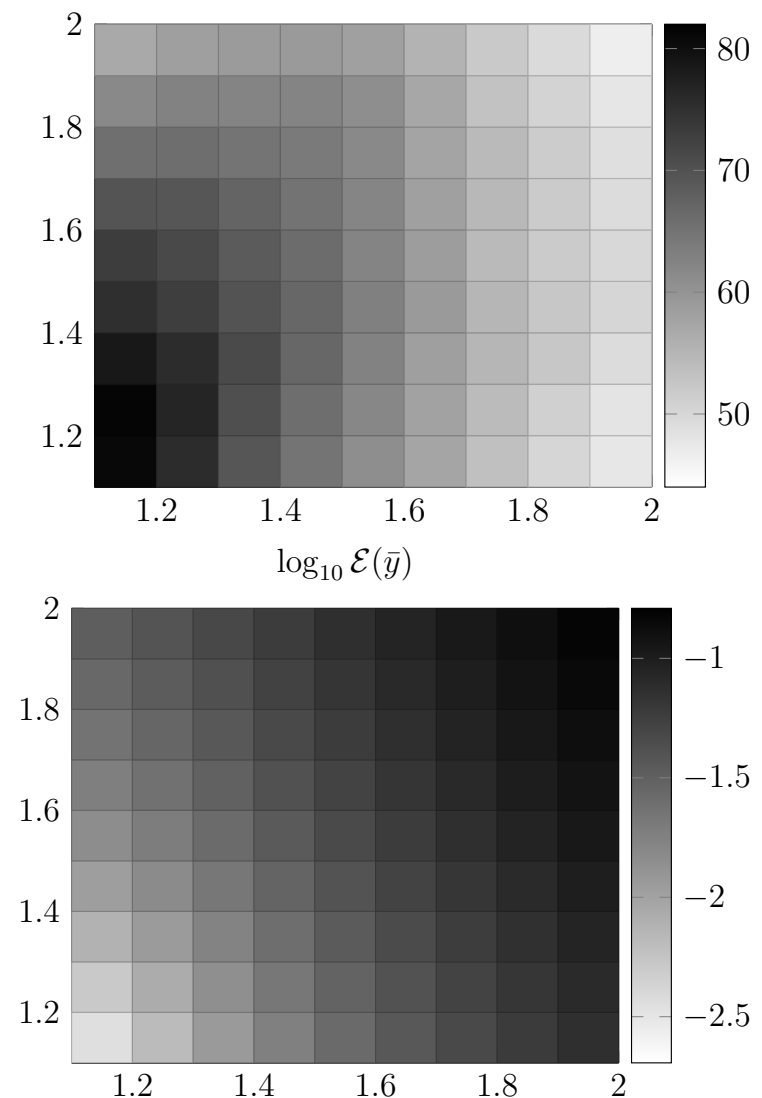

In this example, the MinRES method would be prohibitive computationally due to the large TT ranks, so we investigate only the SC2 approach. In Table 7 we show the performance w.r.t. the grid size. Again, the reference solution $y_{\star}$ is obtained by the SC2 method with accuracy $\varepsilon_{\star}=10^{-8}$. We observe that the behavior is qualitatively the same as for the $2 \mathrm{D}$ problem with incomplete data. We see that the TT ranks stabilize, and the CPU time tends to a linear growth with $l$, but the accuracy of the solution deteriorates proportionally to the condition number of the system matrix, because so does the accuracy of the matrix inversion in the Schur complement. Nevertheless, at moderate grids (e.g. 7) the results are satisfactory, considering the fact that the full problem of size $2^{35}$ with a dense matrix is intractable.

In Fig. 3 we show volumetric plots of the solution, control and Lagrange multiplier vectors at the final time $T=1$, computed at the grid level $l=5$. We see a good agreement with the target solution. An interesting feature is the anisotropic structure of the control. The 1.5-order fractional derivative possesses some properties of the convection first-order operator. In particular, a positive force is exerted on the left side of the center (the peak of the Gaussian function): the (inverse) fractional operator moves it to the right, towards the centered Gaussian function.

\section{Conclusions and outlook}

We have presented numerical algorithms for the optimization of an objective function subject to a fractional differential equation constraint. For this we discussed the discretization of 
Table 6: Variable coefficient test. CPU times (sec.), TT ranks, iterations and errors vs. the grid level $l$.

\begin{tabular}{c|cc|ccc||cc||cc}
$l$ & \multicolumn{2}{|c|}{ SC2 } & \multicolumn{3}{|c|}{ MR } & Full & \multicolumn{2}{|c}{ SC2 } & MR \\
\hline & time & rank & time & rank & iter & time & \multicolumn{2}{|c}{$\mathcal{E}\left(y_{\star}\right)$} \\
3 & 0.6107 & 13 & 5.534 & 47 & 13 & 13.68 & $5.77 \mathrm{e}-7$ & $9.12 \mathrm{e}-5$ \\
4 & 1.5428 & 20 & 37.49 & 235 & 15 & 248.8 & $2.07 \mathrm{e}-6$ & $5.05 \mathrm{e}-4$ \\
5 & 6.2072 & 29 & 2610 & 954 & 20 & 16537 & $9.54 \mathrm{e}-7$ & $7.27 \mathrm{e}-4$ \\
6 & 32.363 & 39 & & - & & - & $2.53 \mathrm{e}-6$ & - \\
7 & 118.62 & 44 & & - & & - & $3.60 \mathrm{e}-5$ & - \\
8 & 311.82 & 45 & & - & & - & $1.62 \mathrm{e}-4$ & - \\
9 & 730.94 & 47 & & - & & - & $2.11 \mathrm{e}-4$ & - \\
10 & 1670 & 48 & & - & & - & $1.44 \mathrm{e}-3$ & - \\
11 & 4309 & 48 & & - & & - & $6.75 \mathrm{e}-3$ & -
\end{tabular}

Table 7: 3D test. Left: CPU times (sec.) and maximal TT ranks of the SC2 method vs. grid level $l$. Right: solution errors.

\begin{tabular}{c|cc||cc}
$l$ & time & rank & $\mathcal{E}\left(y_{\star}\right)$ & $\mathcal{E}(\bar{y})$ \\
\hline 3 & 1.253 & 21 & $4.58 \mathrm{e}-7$ & $1.05 \mathrm{e}-2$ \\
4 & 5.425 & 43 & $2.22 \mathrm{e}-6$ & $8.02 \mathrm{e}-3$ \\
5 & 19.96 & 71 & $9.43 \mathrm{e}-6$ & $6.60 \mathrm{e}-3$ \\
6 & 61.11 & 83 & $7.62 \mathrm{e}-5$ & $8.11 \mathrm{e}-3$ \\
7 & 135.5 & 85 & $4.84 \mathrm{e}-4$ & $9.05 \mathrm{e}-3$ \\
8 & 302.8 & 86 & $2.27 \mathrm{e}-3$ & $9.38 \mathrm{e}-3$ \\
9 & 583.6 & 86 & $9.92 \mathrm{e}-3$ & $1.20 \mathrm{e}-2$ \\
10 & 941.6 & 86 & $5.54 \mathrm{e}-2$ & $4.58 \mathrm{e}-2$ \\
11 & 1132 & 85 & $2.11 \mathrm{e}-1$ & $1.73 \mathrm{e}-1$
\end{tabular}

the differential equation via the well-known Grünwald-Letnikov finite difference method. Using a classical Lagrangian approach we obtained a saddle point system of vast dimensionality representing the first order optimality conditions. We showed that these systems have an inherent tensor product structure. For the efficient solution of these systems we discussed three possible approaches. Two of these methods are variations of a Schur complement approach, and the third is a preconditioned Krylov subspace solver. As the storage requirements are too large for all practically relevant scenarios when using a more fundamental approach, we then introduced algorithms working with a compressed storage format. Namely we utilized a tensor train method, which was also amendable for a tensorized MinREs solver as well as alternating tensor solvers. We then illustrated the performance of these solvers on various setups and showed their competitiveness for vast system dimensions.

For future research in this field, we believe the study of nonlinear problems such as the phase-field equations described above will be of great value. Furthermore, the study of FDEs with uncertain parameters is a challenging and interesting topic. In this setting one can again separate the problem into low-rank objects, as the presented techniques can additionally achieve low-rank solutions even if the spatial dimension is not in tensor form, e.g. discretization via finite elements on possibly irregular domains. We aim to base our approaches for these applications on recent results presented in [9, 7]. 
Figure 3: 3D test. $y$ (top left), $\bar{y}$ (top right), $u$ (bottom left) and $p$ (bottom right) at the final time.
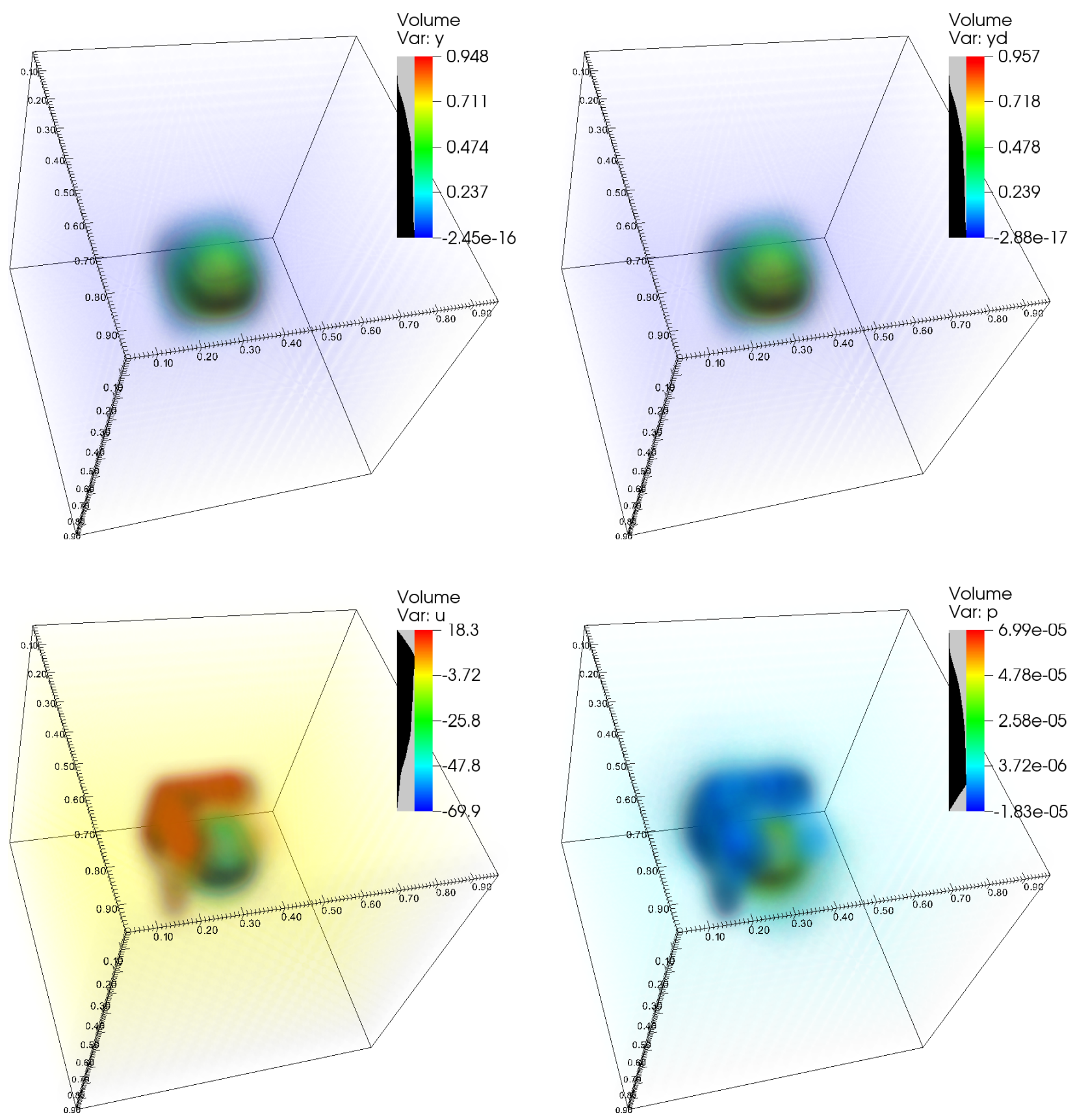

\section{Appendix. Implementation of the TT solvers}

The AMEn Algorithm 2 for solution of linear systems is provided by the procedure amen_solve2.m in the TT-Toolbox, and for the fast approximation of products $A y$ and $A^{\top} y$ within MinRES we used the procedure amen_mv.m.

Despite the progress of the tensor methods, they still require some parameter tuning for better performance. We used the Frobenius norm of the error between two consecutive iterations as an error measure. This is specified by passing the parameters 'trunc_norm' , 'fro' 
to amen_solve2. To address the ill conditioning of $S$ in (18), for the SC1 method we take local_restart $=100$, max_full_size $=2500$ and tol_exit $=5 \varepsilon$. The first two parameters increase the accuracy of the solver for (29), and the third parameter removes unnecessary iterations near a tolerance of $\varepsilon$ by stopping at $5 \varepsilon$. Since the TT ranks of $A^{-1}$ are rather large, we set kickrank $=10$ for (21). This allows to increase the ranks faster and have fewer iterations. The Schur complement $S$ in 20 is also ill-conditioned, and we set local_restart $=100$ for this stage. Finally, in the preconditioning stage of the MinRES method, we start the AMEn algorithm using the given right-hand side as the initial guess, as $\widetilde{A}$ is reasonably close to the identity matrix.

\section{References}

\section{References}

[1] G. Adomian. Solving Frontier Problems of Physics: The Decomposition Method. Kluwer Academic Publishers, 1994.

[2] O. P. Agrawal. A general formulation and solution scheme for fractional optimal control problems. Nonlinear Dynam., 38:323-337, 2004.

[3] T. Akbarian and M. Keyanpour. A new approach to the numerical solution of fractional order optimal control problems. Appl. Appl. Math., 8(2):523-534, 2013.

[4] J. Ballani and L. Grasedyck. A projection method to solve linear systems in tensor format. Numerical Linear Algebra with Applications, 20(1):27-43, 2013.

[5] U. Baur and P. Benner. Factorized solution of Lyapunov equations based on hierarchical matrix arithmetic. Computing, 78(3), 2006.

[6] P. Benner, R.-C. Li, and N. Truhar. On the ADI method for Sylvester equations. J. Computat. Appl. Math., 233(4):1035-1045, 2009.

[7] Peter Benner, Sergey Dolgov, Akwum Onwunta, and Martin Stoll. Low-rank solvers for unsteady Stokes-Brinkman optimal control problem with random data. Submitted, 2015.

[8] Peter Benner and Patrick Kürschner. Computing real low-rank solutions of Sylvester equations by the factored ADI method. MPI Magdeburg Preprint MPIMD/13-05, May 2013.

[9] Peter Benner, Akwum Onwunta, and Martin Stoll. Block-diagonal preconditioning for optimal control problems constrained by PDEs with uncertain inputs. Submitted, 2015.

[10] M. Benzi, G.H. Golub, and J. Liesen. Numerical solution of saddle point problems. Acta Numer., 14:1-137, 2005.

[11] M. Benzi, E. Haber, and L. Taralli. A preconditioning technique for a class of PDEconstrained optimization problems. Adv. Comput. Math., 35:149-173, 2011.

[12] Jessica Bosch and Martin Stoll. A fractional inpainting model based on the vector-valued Cahn-Hilliard equation. Submitted to SIAM Journal on Imaging Sciences, 2015. 
[13] Tobias Breiten, Valeria Simoncini, and Martin Stoll. Fast iterative solvers for fractional differential equations. Submitted, 2014.

[14] Alfonso Bueno-Orovio, David Kay, and Kevin Burrage. Fourier spectral methods for fractional-in-space reaction-diffusion equations. BIT Numerical Mathematics, 54(4):937$954,2014$.

[15] Kevin Burrage, Nicholas Hale, and David Kay. An efficient implicit FEM scheme for fractional-in-space reaction-diffusion equations. SIAM Journal on Scientific Computing, 34(4):A2145-A2172, 2012.

[16] M. Caputo and F. Mainardi. Linear models of dissipation in anelastic solids. Rivista del Nuovo Cimento, 1:161-198, 1971.

[17] WH Deng and Jan S Hesthaven. Local discontinuous galerkin methods for fractional diffusion equations. ESAIM: Math. Model. Num., 47(06):1845-1864, 2013.

[18] K. Diethelm. The analysis of fractional differential equations: an application-oriented exposition using differential operators of Caputo type. Lecture Notes in Mathematics. Springer, 2004.

[19] Kai Diethelm, Neville J Ford, Alan D Freed, and Yu Luchko. Algorithms for the fractional calculus: a selection of numerical methods. Comput. Method Appl. M., 194(6):743$773,2005$.

[20] S. Dolgov and B. Khoromskij. Two-level QTT-Tucker format for optimized tensor calculus. SIAM J. on Matrix An. Appl., 34(2):593-623, 2013.

[21] S. V. Dolgov. TT-GMRES: solution to a linear system in the structured tensor format. Russ. J. Numer. Anal. Math. Model., 28(2):149-172, 2013.

[22] S. V. Dolgov. Tensor product methods in numerical simulation of high-dimensional dynamical problems. notpreprint, University of Leipzig, 2014.

[23] S. V. Dolgov and D. V. Savostyanov. Alternating minimal energy methods for linear systems in higher dimensions. SIAM J. Sci. Comput., 36(5):A2248-A2271, 2014.

[24] V. Druskin and L. Knizhnerman. Extended Krylov subspaces: approximation of the matrix square root and related functions. SIAM J. Matrix Anal. Appl., 19(3):755-771, 1998.

[25] H.C. Elman, D.J. Silvester, and A.J. Wathen. Finite Elements and Fast Iterative Solvers: with Applications in Incompressible Fluid Dynamics. Numerical Mathematics and Scientific Computation. Oxford University Press, New York, 2005.

[26] HW Engl. Discrepancy principles for tikhonov regularization of ill-posed problems leading to optimal convergence rates. Journal of optimization theory and applications, 52(2):209-215, 1987.

[27] A. D. Freed and K. Diethelm. Fractional calculus in biomechanics: a 3D viscoelastic model using regularized fractional-derivative kernels with application to the human calcaneal fat pad. Biomech. Model. Mechanobiol., 5:203-215, 2006. 
[28] V Gafiychuk, B Datsko, and V Meleshko. Mathematical modeling of time fractional reaction-diffusion systems. Journal of Computational and Applied Mathematics, 220(1):215-225, 2008.

[29] Gene H. Golub and Charles F. Van Loan. Matrix Computations. Johns Hopkins Studies in the Mathematical Sciences. Johns Hopkins University Press, Baltimore, MD, third edition, 1996.

[30] R. Gorenflo and F. Mainardi. Fractional calculus: integral and differential equations of fractional order. In A. Carpinteri and F. Mainardi, editors, Fractals and Fractional Calculus in Continuum Mechanics, pages 223-276. Springer, 1997.

[31] L. Grasedyck and W. Hackbusch. A multigrid method to solve large scale Sylvester equations. SIAM J. Matrix Anal. Appl., 29(3):870-894, 2007.

[32] Per Christian Hansen and Dianne Prost O'Leary. The use of the l-curve in the regularization of discrete ill-posed problems. SIAM Journal on Scientific Computing, 14(6):14871503, 1993.

[33] M. Hazewinkel. Encyclopaedia of Mathematics: A-Integral-Coordinates. Springer, 1995.

[34] Rudolf Hilfer, PL Butzer, U Westphal, J Douglas, WR Schneider, G Zaslavsky, T Nonnemacher, A Blumen, and B West. Applications of fractional calculus in physics, volume 5. World Scientific, 2000.

[35] M. Hinze, R. Pinnau, M. Ulbrich, and S. Ulbrich. Optimization with PDE Constraints. Mathematical Modelling: Theory and Applications. Springer-Verlag, New York, 2009.

[36] S. Holtz, T. Rohwedder, and R. Schneider. The alternating linear scheme for tensor optimization in the tensor train format. SIAM J. Sci. Comput., 34(2):A683-A713, 2012.

[37] Kazufumi Ito and Karl Kunisch. Lagrange multiplier approach to variational problems and applications, volume 15 of Advances in Design and Control. Society for Industrial and Applied Mathematics (SIAM), Philadelphia, PA, 2008.

[38] E. Jeckelmann. Dynamical density-matrix renormalization-group method. Phys. Rev. $B, 66: 045114,2002$.

[39] I.S. Jesus, J.A.T. Machado, and J.B. Cunha. Fractional electrical impedances in botanical elements. J. Vib. Control, 14(9-10):1389-1402, 2008.

[40] V. Kazeev, B. Khoromskij, and E. Tyrtyshnikov. Multilevel Toeplitz matrices generated by tensor-structured vectors and convolution with logarithmic complexity. SIAM J. Sci. Comput., 35(3):A1511-A1536, 2013.

[41] V. A. Kazeev and B. N. Khoromskij. Low-rank explicit QTT representation of the Laplace operator and its inverse. SIAM J. Matrix Anal. Appl., 33(3):742-758, 2012.

[42] B. N. Khoromskij. $\mathcal{O}(d \log n)$-Quantics approximation of $N-d$ tensors in highdimensional numerical modeling. Constr. Approx., 34(2):257-280, 2011. 
[43] B. N. Khoromskij and I. V. Oseledets. DMRG+QTT approach to computation of the ground state for the molecular Schrödinger operator. Preprint 69, MPI MIS, Leipzig, 2010.

[44] R.C. Koeller. Applications of fractional calculus to the theory of viscoelasticity. J. Appl. Mech., 51(2):299-307, 1984.

[45] T. G. Kolda and B. W. Bader. Tensor decompositions and applications. SIAM Rev., 51(3):455-500, 2009.

[46] D. Kressner and C. Tobler. Krylov subspace methods for linear systems with tensor product structure. SIAM J. Matrix Anal. Appl., 31(4):1688-1714, 2010.

[47] A. J. Laub. Matrix Analysis for Scientists and Engineers. Society for Industrial and Applied Mathematics (SIAM), 2005.

[48] Fawang Liu, V. Anh, and I. Turner. Numerical solution of the space fractional FokkerPlanck equation. Journal of Computational and Applied Mathematics, 166(1):209-219, 2004.

[49] Mark M Meerschaert, Hans-Peter Scheffler, and Charles Tadjeran. Finite difference methods for two-dimensional fractional dispersion equation. Journal of Computational Physics, 211(1):249-261, 2006.

[50] M.M. Meerschaert and C. Tadjeran. Finite difference approximations for fractional advection-dispersion flow equations. J. Comput. Appl. Math., 172(1):65-77, 2004.

[51] M.M. Meerschaert and C. Tadjeran. Finite difference approximations for two-sided space-fractional partial differential equations. Appl. Numer. Math., 56(1):80-90, 2006.

[52] R. Metzler and J. Klafter. The random walk's guide to anomalous diffusion: a fractional dynamics approach. Physics reports, 339(1):1-77, 2000.

[53] Kenneth S Miller and Bertram Ross. An Introduction to the Fractional Calculus and Fractional Differential Equations. John Wiley \& Sons, 1993.

[54] G. M. Mophou. Optimal control of fractional diffusion equation. Comput. Math. Appl., 61:68-78, 2011.

[55] G. M. Mophou and G. M. N'Guérékata. Optimal control of a fractional diffusion equation with state constraints. Comput. Math. Appl., 62:1413-1426, 2011.

[56] Malcolm F. Murphy, Gene H. Golub, and Andrew J. Wathen. A note on preconditioning for indefinite linear systems. SIAM J. Sci. Comput., 21(6):1969-1972, 2000.

[57] Jorge Nocedal and Stephen J. Wright. Numerical optimization. Springer Series in Operations Research and Financial Engineering. Springer, New York, second edition, 2006.

[58] Ricardo H Nochetto, Enrique Otárola, and Abner J Salgado. A pde approach to fractional diffusion in general domains: a priori error analysis. arXiv preprint arXiv:1302.0698, 2013. 
[59] I. V. Oseledets. Approximation of matrices with logarithmic number of parameters. Doklady Math., 428(1):23-24, 2009.

[60] I. V. Oseledets. Approximation of $2^{d} \times 2^{d}$ matrices using tensor decomposition. SIAM J. Matrix Anal. Appl., 31(4):2130-2145, 2010.

[61] I. V. Oseledets. Tensor-train decomposition. SIAM J. Sci. Comput., 33(5):2295-2317, 2011.

[62] I. V. Oseledets. Constructive representation of functions in low-rank tensor formats. Constr. Approx., 37(1):1-18, 2013.

[63] I. V. Oseledets and S. V. Dolgov. Solution of linear systems and matrix inversion in the TT-format. SIAM J. Sci. Comput., 34(5):A2718-A2739, 2012.

[64] I. V. Oseledets, D. V. Savostyanov, and E. E. Tyrtyshnikov. Linear algebra for tensor problems. Computing, 85(3):169-188, 2009.

[65] N. Özdemir and D. Avci. Optimal control of a linear time-invariant space-time fractional diffusion process. J. Vib. Control, 20, 2014.

[66] John W. Pearson, Martin Stoll, and Andrew J. Wathen. Regularization-robust preconditioners for time-dependent PDE-constrained optimization problems. SIAM J. Matrix Anal. Appl., 33(4):1126-1152, 2012.

[67] John W. Pearson and Andrew J. Wathen. A new approximation of the Schur complement in preconditioners for PDE-constrained optimization. Numer. Linear Algebra Appl., 19:816-829, 2012.

[68] John W. Pearson and Andrew J. Wathen. Fast iterative solvers for convection-diffusion control problems. Electron. Trans. Numer. Anal., 40:294-310, 2013.

[69] I. Podlubny. Fractional Differential Equations: an Introduction to Fractional Derivatives, Fractional Differential Equations, to Methods of their Solution and some of their Applications, volume 198. Access Online via Elsevier, 1998.

[70] I. Podlubny. Fractional Differential Equations. Academic Press, 1999.

[71] I. Podlubny. Matrix approach to discrete fractional calculus. Fract. Calc. Appl. Anal., 3(4):359-386, 2000.

[72] I. Podlubny, A. Chechkin, T. Skovranek, Y.Q. Chen, and B.M. Vinagre Jara. Matrix approach to discrete fractional calculus II: partial fractional differential equations. $J$. Comput. Phys., 228(8):3137-3153, 2009.

[73] I. Podlubny, I. Petraš, B.M. Vinagre, P. O’leary, and L' Dorčák. Analogue realizations of fractional-order controllers. Nonlinear Dynam., 29(1-4):281-296, 2002.

[74] M. R. Rapaić and Z. D. Jeličić. Optimal control of a class of fractional heat diffusion systems. Nonlinear Dynam., 62(1-2):39-51, 2010. 
[75] J. A. Roberts, D. V. Savostyanov, and E. E. Tyrtyshnikov. Superfast solution of linear convolutional Volterra equations using QTT approximation. J. Comput. Appl. Math., 260:434-448, 2014.

[76] T. Rohwedder and A. Uschmajew. On local convergence of alternating schemes for optimization of convex problems in the tensor train format. SIAM J. Num. Anal., 51(2):1134-1162, 2013.

[77] Y. Saad. Iterative Methods for Sparse Linear Systems. Society for Industrial and Applied Mathematics, Philadelphia, PA, 2003.

[78] S.G. Samko, A.A. Kilbas, and O.O.I. Marichev. Fractional Integrals and Derivatives. Gordon and Breach Science Publishers Yverdon, 1993.

[79] U. Schollwöck. The density-matrix renormalization group in the age of matrix product states. Annals of Physics, 326(1):96-192, 2011.

[80] V. Simoncini. A new iterative method for solving large-scale Lyapunov matrix equations. SIAM J. Sci. Comput., 29(3):1268-1288, 2007.

[81] V. Simoncini. Computational methods for linear matrix equations. Technical report, Università di Bologna, March 2013.

[82] D. C. Sorensen and A. C. Antoulas. The Sylvester equation and approximate balanced reduction. Linear Algebra Appl., 351-352:671-700, 2002.

[83] M. Stoll and T. Breiten. A low-rank in time approach to PDE-constrained optimization. to appear in SIAM J. Sci. Comp.

[84] C.R.T. Tarley, G. Silveira, W.N.L. dos Santos, G.D. Matos, E.G.P. da Silva, M.A. Bezerra, M. Miró, and S.L.C. Ferreira. Chemometric tools in electroanalytical chemistry: methods for optimization based on factorial design and response surface methodology. Microchemical Journal, 92(1):58-67, 2009.

[85] F. Tröltzsch. Optimal Control of Partial Differential Equations: Theory, Methods and Applications. American Mathematical Society, 2010.

[86] E. E. Tyrtyshnikov. Tensor approximations of matrices generated by asymptotically smooth functions. Sbornik: Mathematics, 194(6):941-954, 2003.

[87] H. Wang and N. Du. Fast alternating-direction finite difference methods for threedimensional space-fractional diffusion equations. J. Comput. Phys., 258:305 - 318, 2014.

[88] C. Wex, A. Stoll, M. Fröhlich, S. Arndt, and H. Lippert. How preservation time changes the linear viscoelastic properties of porcine liver. Biorheology, 50(3):115-131, 2013.

[89] Steven R. White. Density-matrix algorithms for quantum renormalization groups. Phys. Rev. B, 48(14):10345-10356, 1993.

[90] Qinwu Xu and Jan S Hesthaven. Stable multi-domain spectral penalty methods for fractional partial differential equations. J. Comput. Phys., 257:241-258, 2014.

[91] P. Yi-Fei. Application of fractional differential approach to digital image processing. Journal of Sichuan University (Engineering Science Edition), 39(3):124-132, 2007. 